\title{
Artificial Intelligence in Drug Discovery: Applications and Techniques
}

\author{
Jianyuan Deng, ${ }^{* \dagger}$ Zhibo Yang, ${ }^{\ddagger}$ Iwao Ojima, ${ }^{\circledR}$ Dimitris Samaras, ${ }^{\ddagger}$ and Fusheng \\ Wang ${ }^{\dagger} \ddagger$ \\ $\dagger$ Department of Biomedical Informatics, Stony Brook University, Stony Brook, NY, USA \\ $\ddagger$ Department of Computer Science, Stony Brook University, Stony Brook, NY, USA \\ IDepartment of Chemistry, Stony Brook University, Stony Brook, NY, USA \\ E-mail: jianyuan.deng@stonybrook.edu
}

\begin{abstract}
Artificial intelligence (AI) has been transforming the practice of drug discovery in the past decade. Various AI techniques have been used in many drug discovery applications, such as virtual screening and drug design. In this survey, we first give an overview on drug discovery and discuss related applications, which can be reduced to two major tasks, i.e., molecular property prediction and molecule generation. We then present common data resources, molecule representations and benchmark platforms. As a major part of the survey, AI techniques are dissected into model architectures and learning paradigms. To reflect the technical development of AI in drug discovery over the years, the surveyed works are organized chronologically. We expect that this survey provides a comprehensive review on AI in drug discovery. We also provide a GitHub repository with a collection of papers (and codes, if applicable) as a learning resource, which is regularly updated.
\end{abstract}




\section{Introduction}

Drug discovery is well known as an expensive, time-consuming process, with low success rates. On average, developing a new drug costs 2.6 billion US dollars ${ }^{1}$ and can take more than 10 years. Moreover, the success rate of launching a drug to market from Phase I clinical trial is daunting, less than $10 \% .2$ In the past decade, the practice of drug discovery has been undergoing radical transformations driven by the rapid development in artificial intelligence $(\mathrm{AI}) \cdot \stackrel{3}{{ }^{3} 7}$ Popular applications of AI in drug discovery include virtual screening,, de novo drug design, ${ }^{9}$ retrosynthesis and reaction prediction, $\stackrel{10}{ }$ and de novo protein design, 11 among others, which can be reduced to two categories, i.e., predictive and generative tasks. To power these AI applications, a wide range of AI techniques are involved, with model architectures evolving from traditional machine learning models to deep neural networks, such as convolutional neural networks, recurrent neural networks, graph neural networks and transformers, etc. Learning paradigms also shift from supervised learning to self-supervised learning and reinforcement learning.

In this survey, we focus on the applications and techniques of AI-driven discovery on small-molecule drugs. Biologics (e.g. antibodies, vaccines) are not covered. We first provide an overview of key applications in drug discovery and point out a collection of previously published perspectives, reviews, and surveys. We then introduce common data resources and representations of small molecules. We also discuss existing benchmark platforms for both molecular property prediction and molecule generation. With knowledge on data and representations, the related techniques, including model architectures and learning paradigms, will be elaborated. Finally, we discuss existing challenges and highlight some future directions. By assembling a Github repository ${ }^{1}$ with the surveyed papers (and codes, if applicable), we expect this survey not only provides a comprehensive overview of AI in drug discovery but also serves as a learning resource for researchers interested in the interdisciplinary field.

\footnotetext{
1 https://github.com/dengjianyuan/Survey_AI_Drug_Discovery
} 


\section{Drug Discovery Overview}

In this section, we first go over the definitions of key concepts in drug discovery, mainly on the screening and design of small molecules. Note that AI-powered drug repositioning, which repurposes existing drugs or drug combinations for new indications, is not included. Besides, target identification, exploiting -omics data for its druggability, is also out of the scope and thus not discussed. Rather, we refer the readers to previous publications on drug repositioning $\frac{12[13}{13}$ and -omics data for target identification. 14

Drug discovery ${ }^{15}$ is a project motivated by the situation when there are no drugs for a disease or when existing drugs have limited efficacy and/or severe toxicity. At the earliest stage, an underlying hypothesis needs to be developed that activation or inhibition of a target (e.g., an enzyme, a receptor, an ion channel, etc) results in therapeutic effects for the disease, which involves target identification and target validation. For the selected target, intensive assays will be performed to find the hits and subsequently the leads (i.e., drug candidates), which involves hit discovery, hit-to-lead phase and lead optimization. The drug candidates then enter preclinical studies and clinical trials. If successful, the drug candidate can be launched into market as a medical product to treat the disease.

To accelerate the small-molecule drug discovery, high-throughput screening (HTS) 16117 has been proposed to increase the discovery efficiency since the 1980s, which is a hit-finding approach underpinned by development in automation and the availability of large chemical libraries. A prominent outcome of HTS is the large-scale structure-activity relationship ( $S A R$ ) datasets, which contribute to the chemical databases such as PubChem ${ }^{18}$ and ZINC.19 Various computational techniques have been developed to search the chemical libraries for

potentially active molecules to be tested in subsequent in vitro and in vivo assays, ${ }^{20}$ which is also known as virtual screening (VS). In other words, VS is to identify active molecules using computational approaches, based on knowledge about the target (structure-based VS) or known active ligands (ligand-based VS) to increase the odds of identifying active molecules. 21 For the concept of active molecules, as mentioned above, activation or inhibition of a target 
is the underlying hypothesis to treat a disease, which corresponds to two major classes of drugs with regard to the mechanism of action (MoA), i.e., agonists and antagonists. ${ }^{22} \mathrm{An}$ agonist is a molecule which activates the target to exert a biologic response as its endogenous ligand. On the contrary, an antagonist is a molecule which binds to the target to block the response. Based on more specific effects and mechanisms, agonists can further be classified as partial agonists, inverse agonists, biased agonists. Antagonists include competitive and non-competitive antagonists. To quantify the activity, various assays have been developed to measure affinity (or potency) and efficacy. Affinity is the fraction or extent to which a molecule binds to a target at a given concentration whereas potency is the necessary amount of a molecule to produce an effect of a given magnitude, inversely proportional to the affinity. Efficacy, on the other hand, describes the effect size, such as inhibition of an enzyme to $60 \%$. Common activity measures are summarized in Table 1.

Table 1: Common Measures of Molecule Activity

\begin{tabular}{ll}
\hline Measures & Definition \\
\hline $\mathrm{Kd}$ & Equilibrium dissociation constant \\
$\mathrm{Km}$ & Michaelis constant \\
$\mathrm{Ki}$ & Inhibition constant \\
\hline IC50 & Half maximal inhibitory concentration \\
EC50 & Half maximal effective concentration \\
\hline
\end{tabular}

Nevertheless, sufficient activity is not the only criterion for an ideal drug candidate, which only makes it a ligand. Binding specificity is another concern. ${ }^{23}$ Most often, a molecule can bind to multiple targets and unexpected side effects may arise due to binding promiscuity. Thus, high selectivity is another desired feature. In fact, drug candidates should also satisfy a combination of criteria, $\stackrel{24}{ }$ with optimal physicochemical (water solubility, acid-base dissociation constant, lipophilicity, permeability), pharmacokinetic (absorption, distribution, metabolism, excretion), and pharmacodynamic (activity, selectivity) properties. Other properties considered during compound synthesis include the Synthetic Accessibility Score (SAS) and the Quantitative Estimation of Drug-likeness (QED). SAS is a heuristic score of how hard (10) or easy (1) it is to synthesize a given molecule based on a combination of the molecular 
fragments' contributions. QED is an estimate (0-1) on how likely a molecule is a viable drug candidate. At its core, drug discovery is a multi-objective optimization problem. ${ }^{25}$ Usually, for each property of interest, a predictive model is built to map the molecular structure to the property value with either classification or regression, which is broadly referred to as quantitative structure-activity relationship (QSAR) modeling. ${ }^{[26}$ A more intriguing prospect from QSAR is that these models can be exploited inversely to reveal the structural features underlying the optimal properties to guide drug design from scratch, also known as de novo drug design.

Rather than merely screening existing chemical libraries, $\frac{27}{27}$ drug design takes a step further to explore the vast chemical space, i.e., the space encompassing all possible small molecules ${ }^{28}$ which has an estimated size around $10^{30}-10^{60} \cdot 29$ In drug design, there is a design-make-test-analysis (DMTA) cycle, which consists of iterative organic synthesis and

property assays. ${ }^{3}$ To efficiently navigate the chemical space, quantitative drug design has been proposed since late 1970s. $\frac{30}{{ }^{30}}$ Essentially, drug design epitomizes in two questions: $\frac{31}{1}$ ) "Can molecular properties be deduced from molecular structures?" and 2) "Which structural features are relevant for certain molecular properties?" The former one also underlies the core assumption of VS and the latter is what QSAR tries to answer. In this sense, drug design can be viewed as an extension to VS, and involves both molecular property prediction and molecule generation, which are the major tasks in current AI-driven drug discovery.

\section{Summary of Existing Reviews}

Next, we briefly discuss existing reviews on AI-driven discovery by dividing them into three categories: 1) "General drug discovery review", 2) "Drug discovery in the AI-era", and 3) "Rethinking AI-driven drug discovery". 


\section{General Drug Discovery Reviews}

Many existing papers have covered the general aspects of drug discovery ${ }^{3 / 15}$ and related concepts, such as chemical space, ${ }^{28} \mathrm{VS}$ and HTS, $2132 \sqrt{33}$ optimal properties for drug candidates, ${ }^{24}$ QSAR, ${ }^{26134}$ target prediction, ${ }^{35}$ and computer-aided drug design. ${ }^{27}$ Besides, one prominent challenge in drug discovery is that molecular properties can be highly sensitive to minor structural changes. This is also known as the activity cliffs (ACs), where pairs of structurally similar molecules exhibit significantly different activities. $[36$ [38 We strongly recommend the readers (especially those new to drug discovery) refer to these reviews for a better understanding on drug discovery and recognition of potential pitfalls.

\section{Drug Discovery in the AI Era}

AI has been widely applied in drug discovery. Since the early 2000s, machine learning models, such as random forest (RF), have been exploited for VS and QSAR. ${ }^{39 \mid 40}$ In 2012, AlexNet $\stackrel{41}{ }$ marked the advent of the deep learning era. ${ }^{42}$ Shortly after in the 2012 Merck Kaggle competition, deep neural networks (DNN) outperformed the standard RF model in predicting molecular activities. $\frac{39}{}$ More recently, the success of AI techniques in computer vision and natural language processing has shed more light into drug discovery $4 / 6 \sqrt[43 \mid 44]{4}$ and led to the burgeoning field of deep learning in chemistry. ${ }^{[5}$ In 2019 , potent inhibitors of discoidin domain receptor 1 (DDR1) were discovered in 21 days by researchers from Insilico Medicine. ${ }^{45}$ In 2020, a novel antibiotic candidate against antibiotic-resistant bacteria, halicin, was identified by researchers from MIT. ${ }^{46}$ Note that AI can be applied at different stages in drug discovery, from target identification and validation to drug response determination. ${ }^{6}$ Lead identification, the focus of this survey, involves two fundamental tasks, i.e., molecular property prediction and molecule generation. Molecular property prediction, at the core of VS, is to predict the property value of a molecule given its structure or learned representation, $\frac{47}{}$ which can be served for various purposes, such as drug-target interaction (DTI) prediction,,$\underline{35}$ toxicity prediction $\underline{48}$ and drug-induced liver injury (DILI) prediction, $\frac{49}{49 m o n g}$ 
others. Molecule generation, underlying drug design, involves two levels of tasks: 1) realistic molecule generation, i.e., generating molecules within constraints imposed by the chemical rules, and 2) goal-directed molecule generation, i.e., generating chemically valid molecules with desired properties. $\underline{50151}$

\section{Rethinking AI-driven Drug Discovery}

Despite the promise of AI in drug discovery, pitfalls still exist, which have been widely

discussed. $89 \sqrt[81 / 52,55]{55}$ As opined by Bender et al, $\underline{53154}$ "a method cannot save an unsuitable representation which cannot remedy irrelevant data for an ill thought-through question". To circumvent potential hypes and unrealistic expectations thereof, there is indeed a necessity to take into consideration the hypotheses, the data, the representations, the models, the learning paradigms and moreover, these components as a whole, for any AI-driven application in drug discovery.

\section{Structure of the Survey}

Given the necessity of a clear understanding on both drug discovery applications and AI techniques, this survey starts from general aspects in drug discovery and then moves on to AIdriven drug discovery, covering data resources, molecule representations, model architectures, and learning paradigms. The organization of this survey is depicted in Fig 1 .

Notably, current literature on the AI techniques is often fragmented. More often than not, the rationale behind the choice of a technique is simply because it has not been previously investigated. $\frac{51}{1}$ To gain more insights into the strengths and weaknesses of these AI techniques, we focus on the model architectures and learning paradigms. We also try to present the surveyed works chronologically so as to reflect the technical development over the years. Finally, we highlight existing challenges and future directions (see Section "Discussion"). 


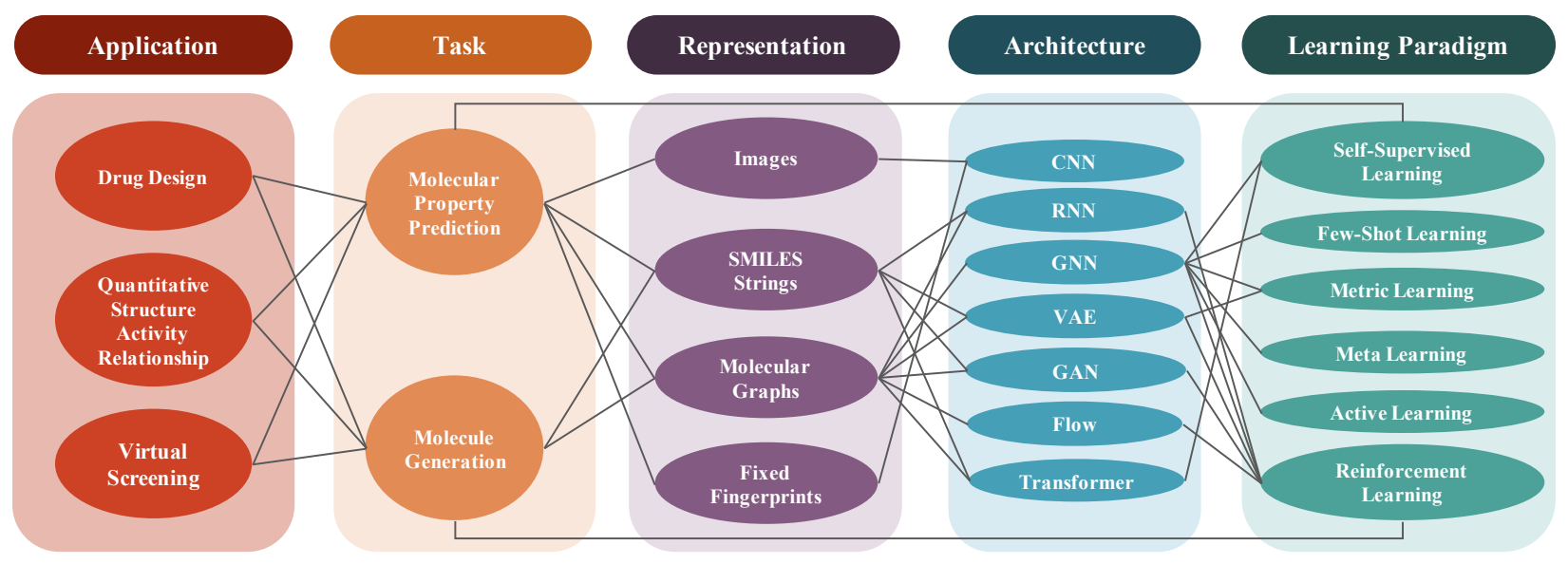

Figure 1: Applications and Techniques of AI in Drug Discovery. The applications of AI in small-molecule drug discovery include virtual screening, quantitative structure-activity relationship and drug design, which can be reduced to two major tasks: molecular property prediction and molecule generation. Small molecules can be represented by fixed fingerprints, molecular graphs, simplified molecular input entry system (SMILES) strings, and images. Various model architectures have been applied on each representation format, including convolutional neural networks (CNN), recurrent neural networks (RNN), graph neural networks (GNN), variational autoencoders (VAE), generative adversarial networks (GAN), normalizing flow models and transformers. Still, challenges exist for the low-data molecular property prediction and goal-directed molecule generation. To tackle these challenges, different learning paradigms have been proposed, such as self-supervised learning for the pretraining-finetuning practice and reinforcement learning for navigating the chemical space search. Other paradigms surveyed here also include few-shot learning, metric learning, meta learning and active learning.

\section{Data, Representation and Benchmark Platforms}

In this section, we first discuss the publicly available data resources. Then, we discuss how small molecules can be represented in machine-readable formats. Lastly, we summarize current benchmark platforms for both molecular property prediction and molecule generation.

\section{Public Data Resources}

With the improvements in HTS and related assays, data on molecular activity and related properties are ever increasing, which contribute to various public data resources. These resources typically provide information on molecular structures, molecular properties and target information, $\sqrt{56}$ as discussed below. 
PubChem ${ }^{57}$ was launched by the National Institutes of Health in 2004. With a collection of chemical information from 750 data sources, PubChem is the largest chemical database. As of August 2020, PubChem contains 111 million unique chemical structures with 271 million activity data points from 1.2 million biological assays experiments. PubChem provides direct download as well as web interfaces for online queries. Notably, PubChem is non-curated ${ }^{56}$ and the bioactivity datasets from PubChem can be highly imbalanced. $\frac{58}{15}$ Researchers may curate the data on their own. For example, Chithrananda et al ${ }^{59}$ recently released a curated dataset of 77 million SMILES strings from PubChem. ChEMBL,,$\frac{60}{2}$ maintained by the European Molecular Biology Laboratory, is another large-scale chemical database. For example, in ChEMBL22 (version 22), there are more than 1.6 million distinct chemical structures with over 14 million activity values. Moreover, ChEMBL is manually curated in a comprehensive manner. ${ }^{56}$ ChEMBL provides downloads in a variety of formats (e.g., Oracle, MySQL or PostgreSQL database) and also allows web application program interface (API) for data retrieval in XML or JSON format. $\stackrel{61}{ }$ Notably, based on ChEMBL, Mayr et al ${ }^{62}$ extracted a large-scale benchmark dataset for target prediction. ZINC, 19 developed by the Irwin and Shoichet Laboratories in UCSF, contains a suite of molecules, annotated ligands and targets as well as the purchasability for over 120 million "drug-like" compounds. ZINC supports direct download from the website and also provides an API for retrieving data. Notably, some subsets of the ZINC database are more commonly used, such as the ZINC-250k $\mathrm{k}^{63}$ and the ZINC Clean Leads collections. $\underline{64}$

In addition to the aforementioned large-scale databases, there are also other data repositories, $\frac{65}{6}$ such as PDBbind, BindingDB, DUD, DUD-E, MUV, STITCH, GLL\&GDD, NRLiST BDB, KEGG, among others. Besides databases mainly derived from preclinical studies, there are also public data resources for the marketed drugs and their effects in human subjects, such as the datasets for adverse drug reactions (ADR) (e.g., DrugBank, SIDER, OFFSIDES and TWO-SIDES) and the datasets for DILI ( e.g., DILIrank). $\underline{66}$ 


\section{Small Molecule Representations}

Molecules are often depicted as Kekulé diagrams with bonds and atoms (Fig 2A). Over the years, machine-readable representations have been developed to enable rapid computation, querying and storage of the molecules. ${ }^{[67}$ Molecules can be represented by the fixed molecular descriptors, which are further categorized by its dimensionality. $\stackrel{56}{ }$ Specifically, there are 0D descriptors for molecules, such as molecular weight (MW), atom number, and atomtype count. 0D descriptors can be directly derived from the empirical formula and barely provide information on how atoms are connected. For example, the empirical formula of alanine, $\mathrm{C}_{3} \mathrm{H}_{7} \mathrm{NO}_{2}$, can also represent lactamide. ${ }^{67}$ To highlight different functional groups, descriptors incorporating more structural information have been proposed, such as fingerprints (Fig 2B). Fingerprints are binary vectors with each dimension in the vector indicating the presence or absence of a particular substructure. Among them, there are 1D descriptors to represent the substituent atoms, chemical bonds, structural fragments, and functional groups. There are also 2D descriptors to represent the atom connectivity and molecular topology, such as 1) Keyed fingerprints - molecular access system (MACCS) keys, 2) Pathbased fingerprints - DayLight fingerprints and 3) Circular fingerprints - extended connectivity

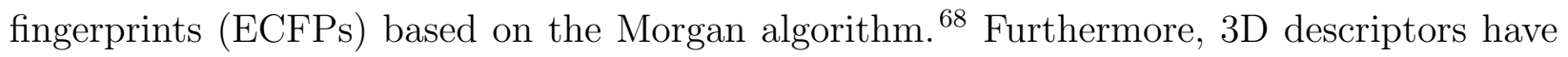
also been developed to encode 3D-structural information like the steric properties, surface area, volume and binding site properties, among others.

Molecular descriptors have greatly boosted the application of computational methods, including machine learning models, in drug discovery. .69170 Nevertheless, these descriptors are fixed and not learnable towards improving model performance. With the advent of the AI era, various deep learning models have paved the way for end-to-end (E2E) predictions, where molecules can be embedded into a continuous latent space without hand-crafted rules. Among them, two major representation formats are molecular graphs and the simplified molecular input entry system (SMILES) strings. 67 

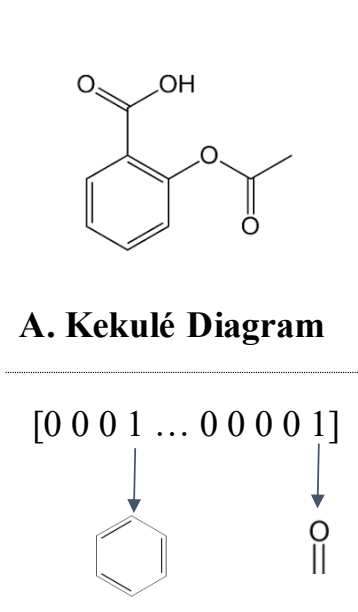

B. Fingerprints

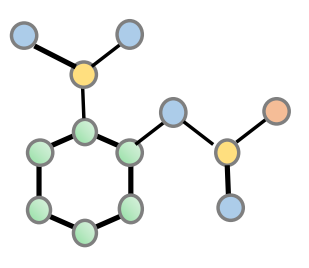

C. Molecular Graph

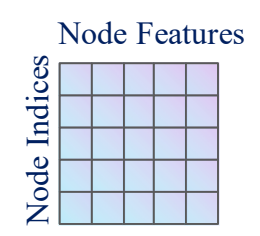

Node Feature Matrix

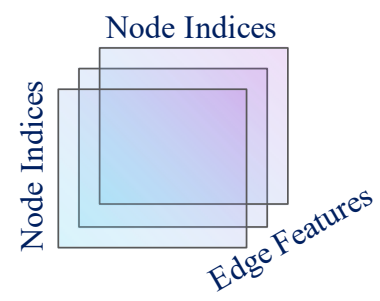

Adjacency Tensor

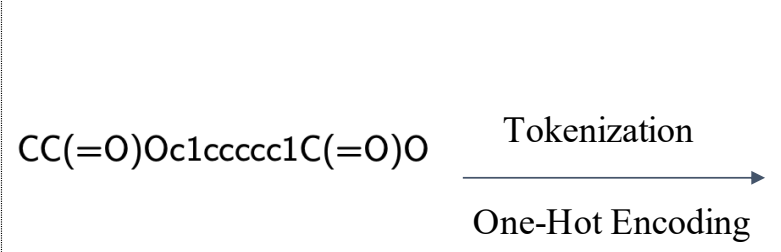

D. SMILES String

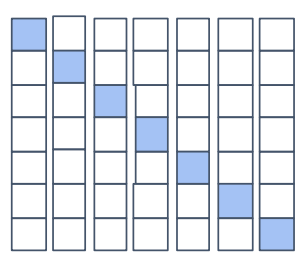

$\mathrm{C}(=\mathrm{O})$ c 1

Figure 2: Illustration of Small Molecule Representations.

\section{Molecular Graphs}

The idea of graph representation is intuitive, where atoms are typically mapped to nodes and bonds to edges. Formally, a graph is defined as $G=(V, E)$, a set of of nodes (atoms) $V$ and a set of edges (bonds) $E$, where $\left(v_{i}, v_{j}\right) \in E$ indicates a bond between atoms $v_{i}$ and $v_{j} .67$ The attributes of atoms are represented by a node feature matrix $X$ and each node $v$ can be represented by an initial vector $x_{v}$ and a hidden vector $h_{v} \in R^{D}$. Similarly, the attributes of bonds can also be represented by an edge feature matrix. Note that both node and edge feature matrices do not directly encode connections. Instead, an adjacency matrix $A$ keeps track of the pairwise connection status. The element of $A, a_{i j}$, if equals to 1 , means that there is a bond connecting node $v_{i}$ and $v_{j}$; otherwise, if $a_{i j}$ equals to 0 , these two nodes are not connected by a bond. Usually, the edge feature matrix and the adjacency matrix are combined to form an adjacency tensor (Fig $2 \mathrm{C}$ ). Common node and edge features ${ }^{71 / 72}$ are summarized in Table 2 ,

One advantage of the graph representations is that they carry more structural information. Besides, molecular graphs as well as the subgraphs can be directly mapped to a chemical (sub-)structure and thus are highly interpretable. ${ }^{73}$ One drawback of the graph 
Table 2: Common Node and Edge Features in Molecular Graphs

\begin{tabular}{lll}
\hline Type & Feature & Notes \\
\hline Node & Atom type & Element type \\
Node & Formal charge & Assigned charges \\
Node & Implicit Hs & Number of bonded hydrogens \\
Node & Chirality & R or S configuration \\
Node & Hybridization & Orbital hybridization: $s p^{x}, s p^{x} d^{y}$ \\
Node & Aromaticity & Aromatic atom or not \\
\hline Edge & Bond type & Single, double, triple or aromatic \\
Edge & Conjugated & Conjugated or not \\
Edge & Stereoisomers & cis (Z) or trans (E) \\
\hline
\end{tabular}

representation, however, is that these matrices require a large amount of disk space for storage and significant memory during computation, which may slow down the efficiency during molecule generation. $\underline{67}$

\section{SMILES Strings}

To accommodate the storage and computation efficiency, molecules are also commonly represented by the SMILES strings. ${ }^{[4}$ In SMILES, an atom is represented by the atomic symbols; for two-character symbols, the second letter will be represented in lower case. Elements in the organic subset, namely B, C, N, O, P, S, F, Cl, Br and I, can be written without brackets whereas for those not included, brackets should be applied with the attached hydrogens and formal charges written inside, such as $[\mathrm{Fe} 2+]$. The lower-case letters represent the atoms in aromatic rings; for instance, $\mathrm{C}$ is used for the normal carbon and $\mathrm{c}$ is used for the aromatic carbon. For bonds, there are single, double, triple and aromatic bonds, represented by the symbols -, =, \# and :, respectively, where single bonds and aromatic bonds are usually omitted. For the branches in a molecule, they are denoted by enclosures in parentheses. To represent the cyclic structure, a single or aromatic bond is first broken down in the ring and then the bonds are numbered in any order with the ring-opening bonds by a digit following the atomic symbol at each ring. Notably one molecule may correspond to multiple SMILES strings. $\frac{67}{6}$ To avoid conflicts, canonicalization methods ${ }^{75}$ have been introduced to ensure only 
one unique SMILES string is designated for the same molecule.

Usually the SMILES string are converted into one-hot vectors before fed into the machine learning models (Fig 2D). ${ }^{76}$ Comparing to the graph representation, SMILES string is less computationally expensive. However, since SMILES strings do not directly encode the atomic connection, there can be a loss of the structural information. ${ }^{77}$ Besides, due to the internal syntax of the SMILES (e.g., ring opening and closure, atom valency), using this linear notations for molecule generation is prone to generate invalid molecules. $\frac{78179}{79}$

\section{Other Representations}

Molecules can also be represented by more sophisticated 3D-atomic coordinates, commonly seen in structure-based VS or QSAR studies. $\frac{80.83}{40}$ Molecular topology, such as bond lengths, bond angles and torsional angles, can also be incorporated $\underline{84}$ Some works have already exploited the 3D-representation to generate molecules. $\frac{85 \mid 86}{1 n}$ addition to the raw 3D coordinates, well-established 3D properties, which capture the molecular conformation, can also be readily utilized for prediction tasks. $\frac{87}{}$ Furthermore, with the advances of computer vision, images of molecular structures (Fig 2A) emerge as another modality to represent molecules. $\underline{88}, 92$

\section{Benchmark Platforms}

To evaluate the performance of molecular property prediction and molecule generation, there are several benchmark platforms, which are discussed below.

As a major benchmark dataset platform for molecular property prediction, MoleculeNet was released by Wu et al in 2018, $\frac{93}{9}$ which includes a set of datasets along with an open-source DeepChem package. ${ }^{94}$ The benchmark datasets cover four categories: 1) Quantum mechanics (QM7, QM7b, QM8, QM9), 2) Physical chemistry (ESOL, FreeSolv, Lipophilicity), 3) Biophysics (PCBA, MUV, HIV, PDDBind, BACE), and 4) Physiology (BBBP, Tox21, ToxCast, SIDER, ClinTox), involving single task or multi tasks. Notably, for molecular property 
prediction, datasets can be highly imbalanced. Thus, when choosing evaluation metrics (Table 3), positive rates should be considered. For instance, AUPRC is favored over AUROC in case of a low positive rate (e.g. less than $2 \%$ ). ${ }^{93}$ With regard to the datset splitting, in addition to the common random split, MoleculeNet also provides other splitting ways, namely, scaffold split, stratified split and time split for different datasets. In other words, for each dataset, the recommended split way varies. For example, for the BACE dataset, since it is for a single target, the scaffold splitting is more suitable, whereas for the PDBind dataset, since the data collection is over a long period, time splitting is recommended to better reflect the actual drug discovery effort over the years.

One limitation of MoleculeNet, however, is that it does not provide explicit training, validation and test folds for the datasets. ${ }^{95}$ To improve reproducibility, the ChemBench package from MolMapNet ${ }^{[96}$ was released recently. MolMapNet also expands the MoleculeNet by adding pharmacokinetics-related datasets, such as PubChem CYP inhibition and liver microsomal clearance data. In addition to the benchmark datasets, Chemprop, ${ }^{71}$ for benchmarking the learned molecular representations, was proposed in 2019, which systematically compared the fixed molecular descriptors (e.g. ECFPs) and the learned molecular representations for molecular property prediction. In Chemprop, models were benchmarked extensively on 19 public and 16 proprietary industrial datasets. As a side note, Chemprop is related to the discovery of halicin. $\underline{46}$

As for benchmarking the molecule generation models, Olivecrona et a ${ }^{[97}$ developed REINVENT in 2017, which is a sequence-based generative model utilizing SMILES strings. REINVENT can be used to execute a range of tasks, such as generating analogues to a query structure and generating ligands for a given target. In 2020, Blaschke et al ${ }^{98}$ proposed the updated version, REINVENT 2.0, as a production-ready tool for drug design. For benchmarking molecule generation utilizing molecular graphs, Mercado et al ${ }^{[72}$ proposed GraphINVENT in 2020. To standardize the assessment for molecule generation, an evaluation framework GuacaMol ${ }^{99}$ was proposed in 2019, which set a suite of tasks for distribution learning and 
goal-directed design. More specifically, the generative model is examined on whether it can reproduce the property distribution of training sets (usually for VS purpose ${ }^{100}$ ), and find the optimal molecule (for multi-objective optimization). A more recent evaluation platform MOSES was released by Polykovskiy et al in 2020, $\underline{64}$ which compiles a list of metrics (Table 3 2 for detecting common issues in generative models, such as overfitting and mode collapse.

Table 3: Commonly Used Evaluation Metrics

\begin{tabular}{llll}
\hline Application & Task & Metric & Purpose \\
\hline Virtual screening & Molecular property prediction & Recall@k & Retrieval \\
Virtual screening & Molecular property prediction & Precision@k & Retrieval \\
Virtual screening & Molecular property prediction & AP@k & Retrieval \\
\hline QSAR & Molecular property prediction & Accuracy & Classification \\
QSAR & Molecular property prediction & Recall & Classification \\
QSAR & Molecular property prediction & Precision & Classification \\
QSAR & Molecular property prediction & AUROC & Classification \\
QSAR & Molecular property prediction & AUPRC & Classification \\
QSAR & Molecular property prediction & MAE & Regression \\
QSAR & Molecular property prediction & RMSE & Regression \\
\hline Drug design & Molecule generation & Validity & Distribution learning \\
Drug design & Molecule generation & Unique@k & Distribution learning \\
Drug design & Molecule generation & Novelty & Distribution learning \\
Drug design & Molecule generation & Diversity & Distribution learning \\
Drug design & Molecule generation & FCD & Distribution learning \\
Drug design & Molecule generation & KL divergence & Distribution learning \\
Drug design & Molecule generation & Scaffold similarity & Goal-directed design \\
Drug design & Molecule generation & Rediscovery & Goal-directed design \\
\hline
\end{tabular}

For instance, validity measures how well a model explicitly captures the chemical rules, such as valency; uniqueness and diversity examine whether the generative model collapses to producing only a limited set of molecules; novelty indicates whether the model is overfitted to just memorize the training examples. Furthermore, Fréchet ChemNet Distance (FCD) is a measure of how close the distributions of the generated set are to the distribution of molecules in the training set. A low FCD value corresponds to similar molecule distributions. Kullback-

\footnotetext{
${ }^{2}$ QSAR: quantitative structure-activity relationship; Recall@k: recall among top k molecules; Precision@k: precision among top $\mathrm{k}$ molecules; AP@k: average precision among top $\mathrm{k}$ molecules; AUROC: area under the receiver-operating characteristic curve; AUPRC: area under the precision-recall curve; MAE: mean absolute error; RMSE: rooted mean square error; Unique@k: uniqueness of the first k valid (generated) molecules; FCD: Fréchet ChemNet Distance; KL divergence: Kullback-Leibler divergence.
} 
Leibler (KL) divergence measures the difference between two probability distributions. When the KL divergence value is small, the generated molecules can well approximate the targeted property in the training set. For goal-directed design, it relies on a formalism where molecules are scored individually based on each pre-defined criterion, such as containing a specific substructure, having certain physicochemical properties or exhibiting similarity or dissimilarity to certain molecules. Consequently, similarity and rediscovery are usually used for evaluation purpose. Specifically, rediscovery assesses if the generative model is able to rediscover a given molecule and similarity evaluates whether the model can generate molecules similar or dissimilar to a given molecule.

\section{Model Architectures}

Prior to the "deep learning" era, traditional machine learning models were widely used in VS. ${ }^{40}$ Pertinent tasks include predictions of drug likeliness, ${ }^{101102}$ physicochemical proper-

ties, ${ }^{1031104}$ pharmacokinetic parameters ${ }^{105}\left[108\right.$ and pharmacodynamic properties ${ }^{109110}$ There are score-based classification models, support vector machines (SVM) 111 and K nearest neighbors (KNN) and probability-based classification models, random forest (RF), ${ }^{112}$ naive bayes (NB), and logistic regression (LR). Despite the success of traditional machine learning models, deep neural networks (DNNs) have outperformed them in a variety of tasks. ${ }^{621113}$

\section{Convolutional Neural Networks}

Convolutional neural networks (CNNs) are mainly used in computer vision to process pixels of data in images. ${ }^{114} \mathrm{In}$ CNNs, there are convolution layers and pooling (i.e. subsampling) layers (Fig 3). On top of these convolution layers and pooling layers, a vector representation is learned by concatenating the feature maps for a final prediction. CNNs share parameters across the filters, which largely reduces the number of parameters to be learned, thereby decreasing memory consumption and increasing computation speed. 


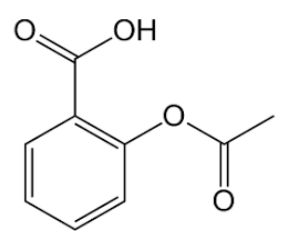

Input Image

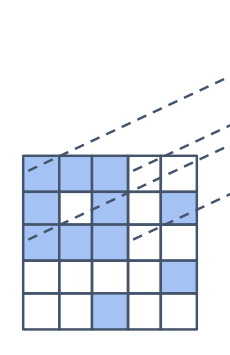

Feature Maps

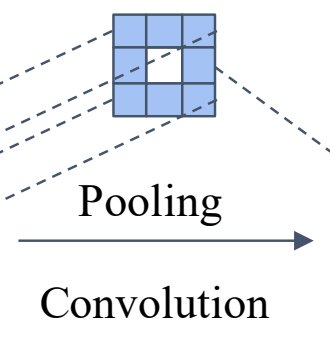

Pooled

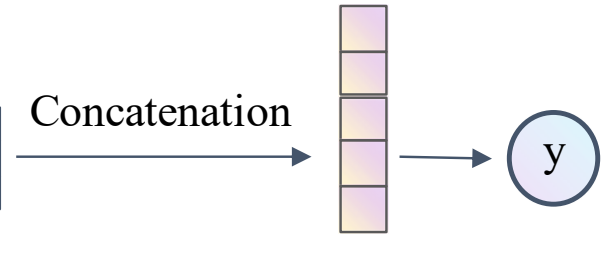

Learned Vector

Figure 3: Illustration of Convolutional Neural Networks.

In drug discovery, CNNs can be applied to elucidate the bioactivity profiles based on microscopy images. 1151116 Moreover, CNNs are also used for molecular property prediction. ${ }^{1171118}$ In 2015, Duvenaud et al ${ }^{[18}$ applied CNNs on circular fingerprints, a refinement of the ECFPs, $\frac{119}{10}$ to create a differentiable fingerprint, which is among the first efforts using data-driven representation learning for molecular property prediction, instead of fixed chemical descriptors. This work has greatly motivated learning molecular representations.

In addition to fingerprints, CNNs can also effectively extract features directly from the images of molecular structure. For instance, Chemception ${ }^{120}$ is trained on the 2D-structural images to predict free energy of solvation and inhibition of HIV replication. Later, Fernández et al ${ }^{88}$ developed Toxic Colors, a framework for toxicity classification with the images as input. Cortes-Ciriano et al ${ }^{90}$ further extended existing CNNs architectures (e.g. AlexNet,, 1$]$ DenseNet-201, $\frac{121]}{\sqrt{121}}$ ResNet152 ${ }^{122}$ and VGG-19 ${ }^{123]}$ ) to Kekulé structure images for molecular property prediction, also known as KekuleScope. The experimental results of KekuleScope showed that CNNs on images as input can achieve comparable performance to RF and DNNs on ECFPs. Meyer et al ${ }^{[89}$ also predicted the MeSH-therapeutic-use classes based on compound images, which outperformed previous predictions based on transcriptomic data. More recently, Rifaioglu et al ${ }^{91}$ proposed a large-scale DTI prediction system, DEEPScreen. Indeed, molecular property prediction with images as input are closely related to the progress in computer vision, which also prompts automatic extraction of chemical structures from literature and patents. ${ }^{921124}$ The chemical structure recognition model can be further inte- 
grated with models in natural language processing, such as DECIMER ${ }^{125}$ and DECIMER $1.0,92$ which are able to translate the bitmap images of a molecule into a SMILES string, as an image captioning task. $\frac{126}{12}$

\section{Recurrent Neural Networks}

Recurrent neural networks (RNNs) are mainly used for processing sequential data. $\frac{114}{2 N N s}$ allow the connection among neurons in the same hidden layer to form a directed cycle (Fig 4A), thereby enabling the use of sequential input, such as language modeling $\frac{127}{12}$ and music generation. $\frac{128}{1 f}$ unfolded in time steps, RNNs can be seen as a very deep feed-forward networks where all layers sharing the same weights. However, the long-term dependency of RNNs makes it difficult to learn the parameters due to the gradient explosion or vanishing problem. ${ }^{114}$ As a result, long short-term memory (LSTM) 129 and gated recurrent unit $(G R U), 130$ two variants of the vanilla RNN, have been developed to augment the network with a memory module. Different from CNNs' operation on images, RNNs mainly take the SMILES strings as input for molecular property prediction and molecule generation. As discussed in Section "Small Molecule Representation", the characters in a SMILES string are firstly converted into one-hot vectors (Fig 2) and then sequentially fed into RNNs, with a hidden vector to be updated at each step. For molecular property prediction, RNNs generate a final output after all steps are taken. For example, SMILES2Vec ${ }^{131}$ uses RNNs to learn features from SMILES and predicts a wide range of chemical properties. Mayr et al ${ }^{62}$ proposed SmilesLSTM to perform DTI prediction, which outperformed traditional machine learning models.

RNNs can also be applied for molecule generation, similar to language models for text generation. ${ }^{97|100| 132}$ More specifically, RNNs generate output at each step in an auto-regressive manner (Fig $4 \mathrm{~B})$, where the output is dependent on the input from previous steps. Based on the input from the current step and prior steps, RNNs output a probability distribution over all possible tokens, from which a token is sampled as the output of the current step and will 


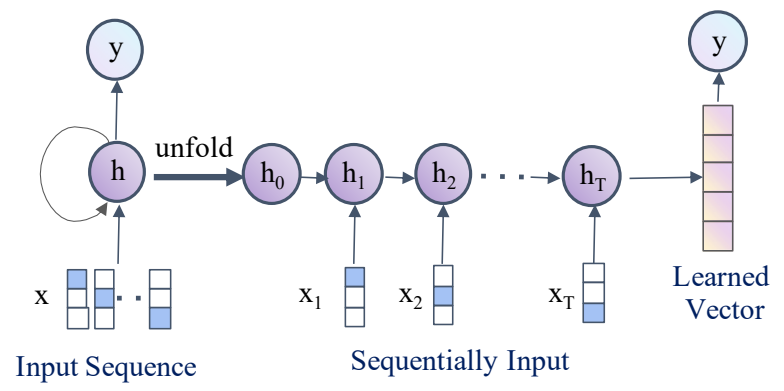

\section{A. Recurrent Neural Networks in Prediction Mode}

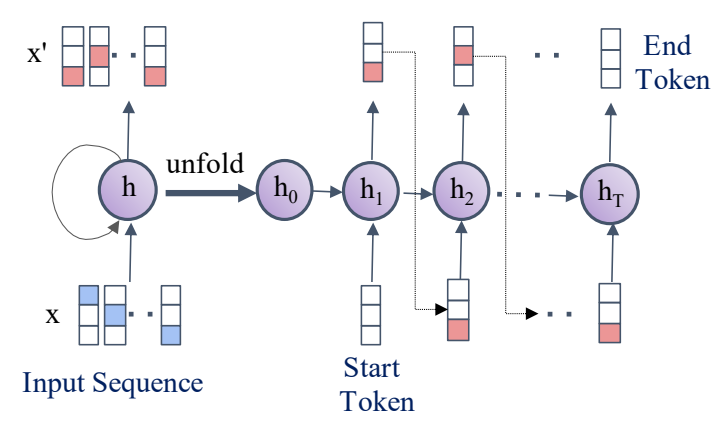

B. Recurrent Neural Networks in Generation Mode

Figure 4: Illustration of Recurrent Neural Networks.

be used to predict the next token. However, due to the syntax of the "SMILES language" such as the ring opening-closure and the matched brackets, regular RNNs, including LSTM and GRU, cannot capture the algorithmic patterns of the sequence well owing to their inability to count. ${ }^{[79}$ As a result, the generated SMILES strings very often violate the chemical rules and become invalid. Thus, a memory-augmented version, Stack-RNN, ${ }^{79 \mid 133}$ was developed to alleviate the validity issue for SMILES-based molecule generation. Another solution for this problem is to adopt bidirectional RNNs, such as the bidirectional LSTM.134135

In addition to the SMILES strings, RNNs can also be applied on molecular graphs for generation purpose.$^{136}[139]$ For example, You et al ${ }^{136}$ proposed GraphRNN to generate molecular graphs in an autoregressive manner, decomposing it as a process into generating a sequence of node and edge formations conditioned on the graph structure generated so far. Nonetheless, generating molecular graphs with RNNs requires a full trajectory of the graph generation, which tends to forget the states of initial generation steps quickly. Later You et al proposed GCPN and ${ }^{140}$ designed the graph generation procedure as a Markov Decision Process (MDP), which only needs the intermediate state to generate the graph. Notably, RNNs can also be components of more complicated generative models, such as variational autoencoders $^{78 \mid 141}$ and generative adversarial networks. $142[143$ 


\section{Graph Neural Networks}

CNNs and RNNs are usually applied on data represented in the Euclidean space. In recent years, graph neural networks (GNNs) are gaining popularity to model data represented in graphs with a set of nodes and edges. ${ }^{144}$ GNNs can handle node-level (e.g., node classification), edge-level (e.g., link prediction) and graph-level (e.g., graph regression) tasks, with neighborhood aggregation, pooling and readout operations. Small molecules, when represented as molecular graphs ( $\mathrm{Fig} 2 \mathrm{2} \mathrm{C})$, are naturally appealing to the application of GNNs for both molecular property prediction and molecule generation tasks (see Section "Molecular Graphs)".

Two major types of GNNs are convolutional GNNs (ConvGNNs) and recurrent GNNs. 144 In the recurrent GNNs, node representation is learned via some recurrent neural architectures, such as the graph gated neural network (GGNN). ${ }^{145}$ On the other hand, ConvGNNs generalize the convolution operation from grid data to graph data and can stack multiple graph convolutional layers to extract high-level node representations. ConvGNNs play a central role in building up many other complex GNNs, which can be further categorized into two subtypes: 1) Spectral-based: ChebNet, $\frac{146}{14}$ graph convolutional network (GraphConv) ${ }^{147}$

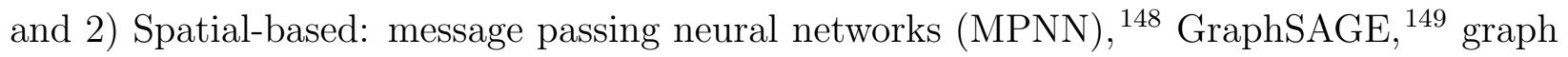

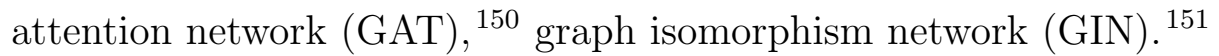

In drug discovery, GNNs are often exploited for molecular property prediction (Fig $5 \mathrm{~A}$ ). For instance, Kearnes et a $\mathrm{a}^{152}$ developed Weave to perform graph convolutions on molecular graphs for representation learning, where the graph convolutions, nonetheless, did not outperform the fingerprint-based models back in 2016. Later, Gilmer et al ${ }^{148}$ proposed MPNN as a unified framework for quantum chemical properties prediction. MPNN has two phases in the forward pass, namely, message passing and readout. During message passing, for each atom, feature vectors from its neighbors are propagated into a message vector wherein the hidden vector for the atom is updated by the message vector. A readout function is used to aggregate the feature vectors into a graph feature vector, which is then passed to a fully con- 


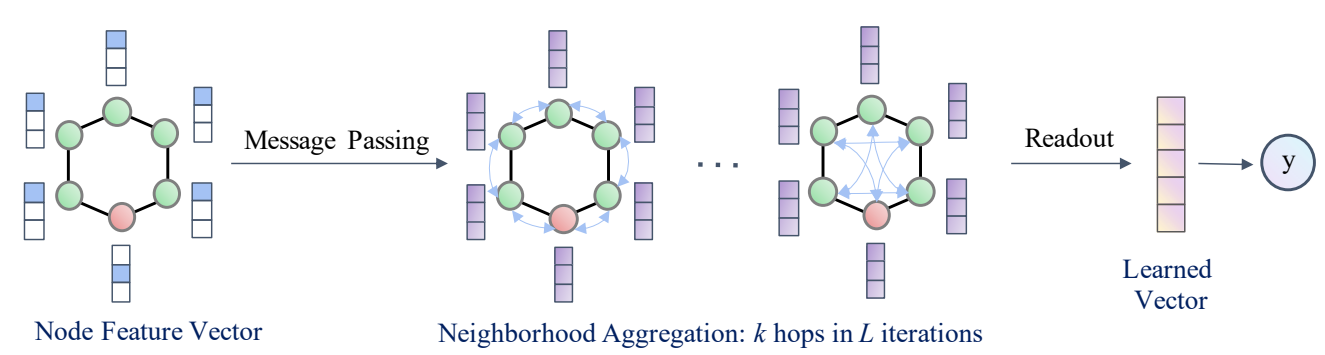

A. Graph Neural Networks in Prediction Mode

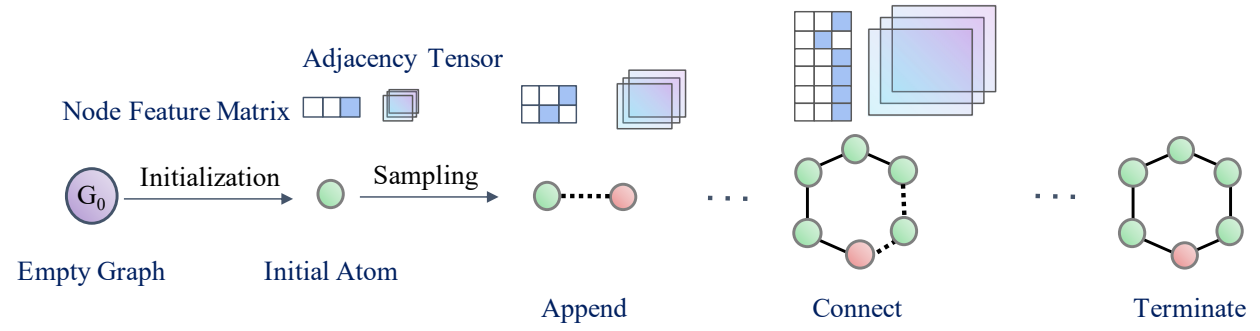

B. Graph Neural Networks in Generation Mode

Figure 5: Illustrations of Graph Neural Networks.

nected layer for downstream predictions. Yang et al ${ }^{71}$ then expanded MPNN into directed MPNN (D-MPNN), which uses messages associated with directed edges (bonds) instead of nodes (atoms) used in MPNN, thereby preventing repeated message passing from the same node. Notably, Yang et al ${ }^{71}$ also introduced a practice to concatenate the 200 global molecular features calculated by RDKit ${ }^{153}$ with the learned features by D-MPNN for downstream predictions, also adopted in later works. ${ }^{63}$ Xiong et al ${ }^{177}$ integrated graph attention mechanism into GNNs and developed Attentive FP, which is able capture topologically adjacent atoms' interactions for improved molecular property prediction. More recently, Withnall et al ${ }^{154}$ also made augmentations to the MPNN and proposed attention MPNN (AMPNN) and edge memory neural network (EMNN) for physicochemical property prediction. So far, GNNs have been widely applied for molecular property prediction. More examples include

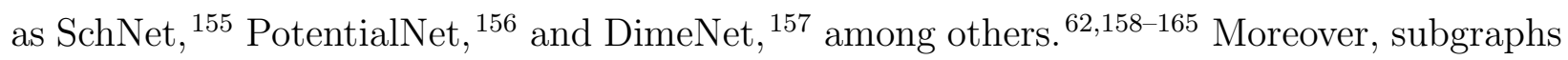
can directly map to molecular substructures, which also improves interpretability. $\frac{77 \mid 166}{168}$

Partly encouraged by the superior performance of GNNs for molecular property prediction, GNNs are also exploited for molecule generation (Fig 5 B). As mentioned above, RNNs 
can be used to generate molecular graphs, which, nevertheless, needs to store a full trajectory for the graph generation process and tends to forget initial states. ${ }^{140}$ In 2018 , Li et al $\frac{138}{138}$ developed a conditional graph generator, MolMP, which does not involve atom-level recurrent units. MolMP models the graph generation as a MDP problem, where the action to grow graph only depends on its current state. There are three actions in total - append, connect and terminate, the sampling process of which is parameterized by a neural network. Experimental results show that MolMP outperforms SMILES-based molecule generation in a variety of evaluation metrics, especially the validity. GNNs-based molecule generation can be used in common drug design applications such as designing molecules with certain scaffolds, presumably due to more straightforward mapping to chemical substructure with the graph representation. Furthermore, since molecule generation is usually driven by certain desired properties, reinforcement learning (see Section "Learning Paradigms"), therefore,

is often integrated with GNNs for goal-directed drug design. Examples include GCPN, 140 MolDQN, $\stackrel{169}{ }$ DeepGraphMolGen, $\frac{170}{2}$ and MNCE-RL. $\stackrel{171}{ }$ For more practical issues on GNNs for molecule generation, such as generation schemes (single-shot vs iterative) and computation, we refer the readers to the guide by Mercado et al. $\frac{51}{51}$

\section{Variational Autoencoders}

Variational autoencoders (VAEs), a class of powerful probablistic generative models, were first introduced by Kingma et al ${ }^{172}$ in 2013. VAEs, consisting of an encoder $E$ and a decoder $D$. The encoder maps high-dimensional data into a low-dimensional, continuous latent space (Fig 6). Compared to common autoencoders, the latent space is regularized to be organized, ideally, through the KL divergence. In addition to reconstruction, VAEs approximate a probability distribution, which can be sampled for generation purpose. Thus, given input $x$, the parameters of VAEs are optimized by minimizing the reconstruction loss and the KL divergence: 173

$$
\|x-D(E(x))\|^{2}+K L\left(N\left(\mu_{x}, \sigma_{x}\right), N(0,1)\right),
$$


which is equivalent to maximize the evidence lower bound (ELBO). In Equation 1, $N(0,1)$ denotes the unit normal distribution; $\mu_{x}$ and $\sigma_{x}$ are learnable parameters, representing mean and variance of a Gaussian distribution.

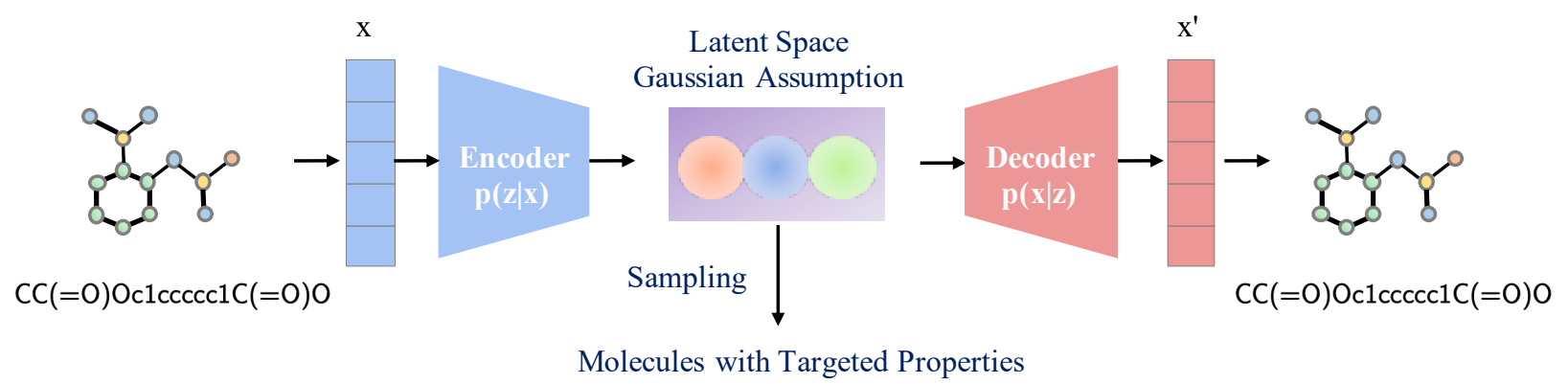

Figure 6: Illustration of Variational Autoencoders.

VAEs can be applied on SMILES strings for molecule generation. For example, GómezBombarelli et al ${ }^{78}$ developed a VAE model for automatic molecule design, where a pair of deep neural networks (i.e. an encoder and a decoder) is trained as an autoencoder to convert the input SMILES strings into a continuous vector representation. To train the autoencoder, a reconstruction loss is adopted in attempt to reproduce the original SMILES string. However, the ultimate goal is not to merely reconstruct the input. Rather, the autoencoder aims to learn a compact representation for the molecules. Thus, a constraint is applied in the autoencoder by jointly training a physical property regression model to organize the VAE's latent space subjected to the property value, which can be used to sample molecules towards the desired property value. Partly due to the syntax of SMILES, the latent space learned by the autoencoder can be sparse and contain large "dead areas", which correspond to invalid molecules. Therefore, VAEs with a focus on the syntax for valid molecule generation are proposed later, such as GrammarVAE ${ }^{174}$ and syntax-directed VAE. ${ }^{175}$ Other related works also include semi-supervised VAE (SSVAE) for continuous output, ${ }^{176}$ conditional VAE (CVAE), ${ }^{177}$ constrained graph VAE (CGVAE), ${ }^{178}$ NeVAE, ${ }^{179}$ GTM VAE ${ }^{141}$ and CogMol..$^{180}$ A variant of the VAE is the adversarial autoencoder (AAE), ${ }^{181}$ which replaces the KL divergence with an adversarial objective. More specifically, the Gaussian distribution assumption 
as a prior on the latent representations for KL-divergence computation is replaced by other priors, i.e., an additional discriminator is added to force the encoder generates latent representations in a specific distribution (e.g. a uniform distribution). AAEs can also be used for molecule generation, $\frac{182}{184}$ which can improve reconstruction and the validity of generated molecules.

VAEs can also be applied on the graph representations for generation purpose. In 2018, Simonovsky et al $\frac{185}{18}$ proposed a VAE framework (GraphVAE) to generate molecular graphs. Their main idea is to output a probabilistic fully-connected graph and use a standard graph matching algorithm to align it to the ground truth. Jin et al $\frac{186}{18}$ developed the junction tree VAE (JT-VAE). In JT-VAE, a molecular graph is first mapped into a junction tree via a tree decomposition algorithm and the junction tree then undergoes the VAE's encodingdecoding process. The learned latent space of the junction tree can be used to search for substructures, which then assemble into molecules with specific properties. A prominent merit of JT-VAE is that the validity of all generated molecules can be guaranteed. Ma et al $\frac{187}{187}$ also proposed a regularization framework for VAEs (Regularized VAE) that regularize the output distribution of the decoder, thereby improving the validity. Later, Kajino et al ${ }^{188}$ developed molecular hypergraph grammar VAE (MHG-VAE), where a molecular graph is described as a hypergraph and the grammar VAE ${ }^{174}$ is trained by inputting the grammar for sequence production of the hypergraph. In 2019, Kwon et al ${ }^{[189}$ developed a non-autoregressive graph VAE and incorporated three additional learning objectives into the model, namely, approximate graph matching, reinforcement learning, and auxiliary property prediction, which is able to generate valid and diverse molecular graphs with various constraints. Moreover, graph-based VAEs can also embrace a strategy for drug design with the ability to retain a particular scaffold (i.e., substructure), such as the ScaffoldVAE. 190

One drawback of VAEs, nonetheless, is that the set of substructures by partitioning molecules can be quite large. Consequently, the iterative prediction of which substructure to add can be inaccurate, especially for infrequent substructures. To address this challenge, Fu 
et al ${ }^{191}$ proposed a novel strategy, CORE, by combining scaffolding tree generation and adversarial training. Besides, the computational cost increasing with the number of nodes in a graph is another major challenge here, limiting the application to larger molecules. In 2020, Kwon et al ${ }^{192}$ proposed a compressed graph representation to alleviate computational complexity while maintaining the validity and diversity of generated molecules. More recently, Jin et al ${ }^{193}$ developed hierarchical graph VAE (HierVAE) which can employ larger and more flexible graph motifs as building blocks for molecules. More specifically, the encoder produces a multi-resolution representation for each molecule in a fine-to-coarse fashion, from atoms to connected motifs while the autoregressive coarse-to-fine decoder adds one motif at a time. Notably, HierVAE can even be used to generate polymers.

\section{Generative Adversarial Networks}

Generative Adversarial Networks (GANs), developed by Goodfellow et al ${ }^{194}$ in 2014, have made remarkable achievements in generating realistic synthetic samples. GANs consist of a generative model $G$, and a discriminative model $D$ (Fig 7 ). The generator aims to generate new data points from a random distribution whereas the discriminator aims to classify whether the generated samples are from the training data distribution or from the generator. GANs can be trained by the min-max loss, which alternatively optimizes the generator and the discriminator using a min-max objective:

$$
\min _{G} \max _{D} \mathcal{L}(G, D)=\mathbb{E}_{x \sim p_{x}}[\log (D(x))]+\mathbb{E}_{z \sim p_{z}}[\log (1-D(G(z)))]
$$

where $p_{x}$ and $p_{z}$ denote the distribution of the real data $x$ and the noise prior $z$.

GANs can be applied to SMILES strings for molecule generation. In 2017, Guimaraes et

al ${ }^{142}$ developed objective-reinforced GANs (ORGAN), built upon SeqGAN, ${ }^{195}$ to generate molecules in SMILES strings while also optimizing several domain-specific metrics. The generator is based on LSTM, which is modeled as a stochastic policy in a reinforcement learning 


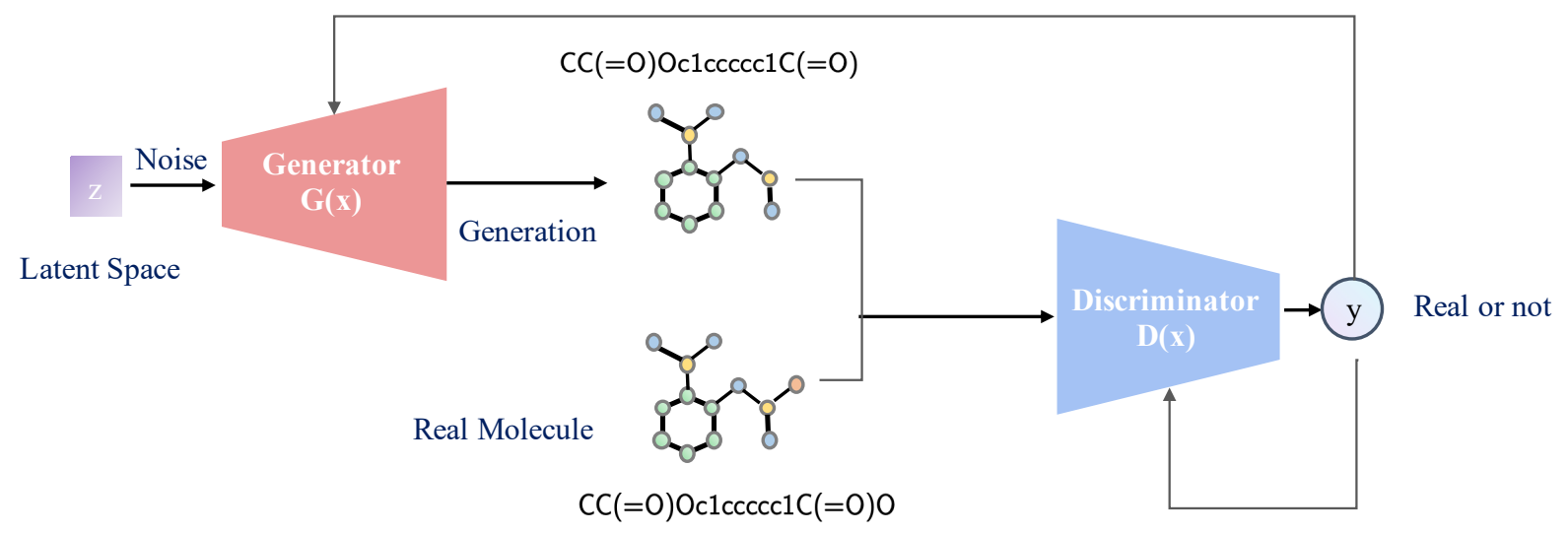

Figure 7: Illustration of Generative Adversarial Networks.

setting (more details in Section "Learning Paradigms"), whereas the Wasserstein loss is used train the discriminator (a CNN model). Experimental results showed that the generated molecules exhibit drug-like structures as well as improvement in the evaluation metrics. Shortly after, an objective-reinforced GANs for inverse-design chemistry (ORGANIC) ${ }^{143}$ was developed based upon ORGAN. ORGANIC can generate molecules with biased distribution towards certain attributes for both drug discovery and material design. In 2018, Putin et $\mathrm{al}^{[196}$ presented a reinforced adversarial neural computer (RANC) framework, which also combines GANs and RL. The generator of the RANC framework is a differentiable neural computer (DNC) with an explicit memory bank, instead of the LSTM model in ORGANIC and ORGAN. This is because the generation of discrete data using RNNs, particularly, LSTM with maximum likelihood estimation, can suffer from the so-called "exposure bias", i.e., missing salient features of the data. RANC outperforms ORGANIC, as measured by several metrics: number of unique structures, passing medicinal chemistry filters (MCFs), Muegge criteria and high QED scores. RANC is able to generate molecules that match the distributions of the key chemical features/descriptors (e.g., MW, logP) and lengths of the SMILES strings from the training set.

GANs can also be applied on molecular graphs for molecule generation. In 2018, De Cao et al ${ }^{197}$ developed MolGAN, an implicit, likelihood-free generative model for small molecular graph generation, which circumvents the expensive graph matching procedures. $\frac{[85}{18}$ Moreover, 
they adapted the GANs to enable direct operation on molecular graphs. RL is also integrated to encourage the generation of molecules towards desired properties. Experimental results on the QM9 dataset showed that MolGAN is able to generate nearly $100 \%$ valid molecules, which outperforms ORGAN in validity. One drawback of MolGAN is its susceptibility to mode collapse, i.e., repeated samples being generated multiple times, leading to low uniqueness. $\frac{198}{1}$

\section{Normalizing Flow Models}

In addition to the RNNs, VAEs and GANs, another major class of generative models is the normalizing flow models. $\frac{199}{199}$ Representative works include the Non-linear Independent Component Estimation model (NICE), 200 the Real-valued Non-Volume Preserving model (RealNVP), 201 and the Glow model, among others. In NICE, Dinh et al 200 introduced tractable calculation for reversible transformations, which are also known as the affine coupling layers underlying the flow models. The basic idea of flow models is to learn an invertible mapping between complex distributions and simple prior distributions (Fig 8). By exploiting exact and tractable likelihood estimation for training, flow models enable efficient one-shot inference and $100 \%$ reconstruction of the training data. 51

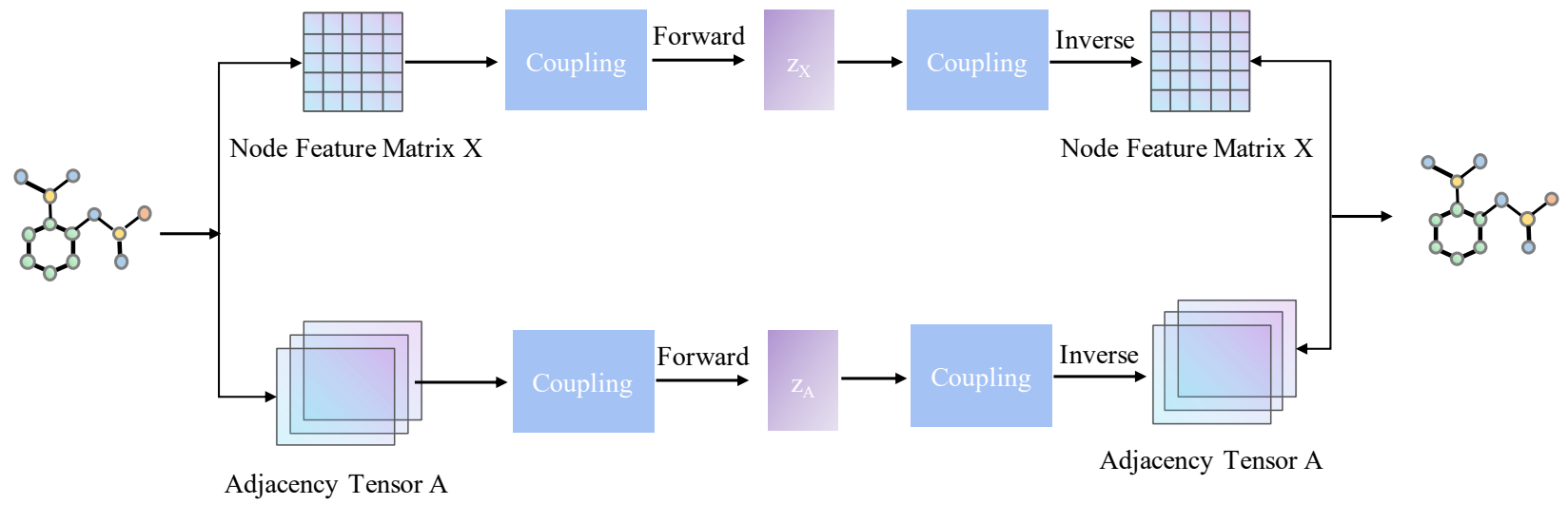

Figure 8: Illustrations of Flow Models.

In drug discovery, flow models have been applied to generate molecules, mainly on molecular graphs. In 2019, Madhawa et al ${ }^{202}$ developed GraphNVP, the first flow model for molecular graph generation. In GraphNVP, the graph generation is decomposed into two steps, 
i.e., generation of an adjacency tensor and generation of node attributes, which yields the exact likelihood maximization on the graph with two reversible flows. GraphNVP is able to generate valid molecules with minimal duplicates. The learned latent space can be further exploited to generate molecules with desired properties. Honda et al ${ }^{203}$ also developed an invertible flow model for molecular graph generation based on residual flows, also known as graph residual flow (GRF), which enables more flexible and complex non-linear mappings than the traditional coupling flows. Experimental results showed that GRF can achieve comparable performance with GraphNVP, while having much less parameters to learn. Notably, GraphNVP 202 and GRF ${ }^{203}$ generate molecular graphs in a single-shot manner, ${ }^{51}$ which may lead to low validity, nevertheless. Consequently, a sequential iterative graph generation manner is proposed for flow models. For example, GraphAF, $\stackrel{204}{2}$ an autoregressive flow-based model, adopts an iterative sampling process to leverage chemical domain knowledge, such as valency checking in each step. With the integration of chemical rules, GraphAF is able to generate molecules of $100 \%$ validity. Moreover, its training process is significantly faster than GCPN. ${ }^{140}$ GraphAF can be further finetuned with RL, which achieves better performance on molecular property optimization compared to JT-VAE ${ }^{186}$ and GCPN. ${ }^{140}$ MoFlow, later developed by Zang et al, ${ }^{205}$ applies a validity correction to the generated graph, which not only enables efficient molecular graph generation in a single-shot manner, but also guarantees the chemical validity. The continuous latent space learned via encoding the molecular graphs can be further used to generate novel and optimized molecules during the decoding process towards desired properties. More recently, Luo et al ${ }^{206}$ developed GraphDF, which, on the contrary, aims to learn a discrete latent representation with the flow models and capture the original discrete distribution of the discrete graph structures without adding real-valued noise. For molecule generation, GraphDF sequentially samples the discrete latent variables and maps them to new nodes and edges via invertible transforms. The discrete transforms can circumvent the cost of computation while also achieving state-of-the-art performance in random molecule generation, property optimization and constrained optimization tasks. 
For the flow-based generative models, the most prominent feature is that they are able to exactly reconstruct all the input data without duplicates due to the precise likelihood maximization, which can be an important complement for molecule generation. In particular, when the molecular property is highly sensitive to minor structural changes, i.e., activity cliffs, $\frac{36137}{2}$ a replacement of a specific atom (node) might be needed. In other words, flow models can offer more precise modifications on existing molecular structures.

\section{Transformers}

RNNs have been widely applied to handle sequential input. However, RNNs can suffer from the gradients explosion or the vanishing problem. $\frac{114}{10}$ In 2017, a seminal work "Attention is all you need" proposed a novel transformer architecture, built with the self-attention mechanism. ${ }^{207}$ Transformers have now become the de facto standard in powerful language models, such as GPT, ${ }^{208}$ BERT, $\stackrel{209}{2}$ GPT-2, ${ }^{210}$ RoBERTa, $\stackrel{211}{21}$ and GPT-3,, 212 and even in advanced computer vision models, such as DETR ${ }^{213}$ and Vision Transformer. ${ }^{214}$ Unlike RNNs, transformers forfeit recurrent connections. By adopting positional embedding, transformers are even better at dealing with long sequences. ${ }^{207}$

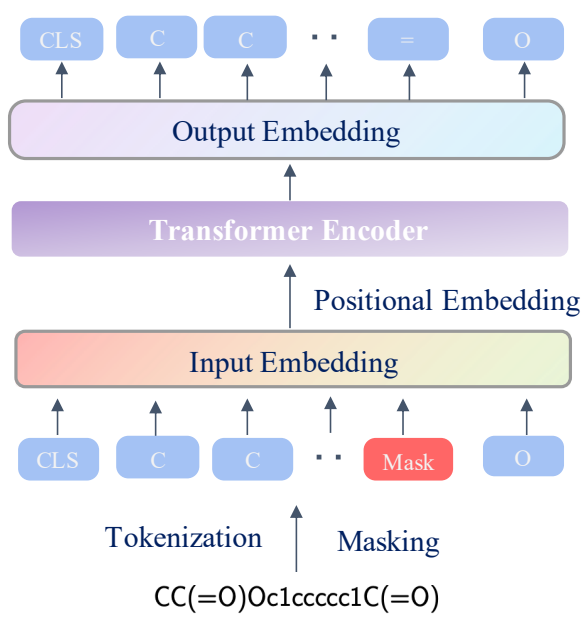

A. Masked Language Modeling on SMILES Strings

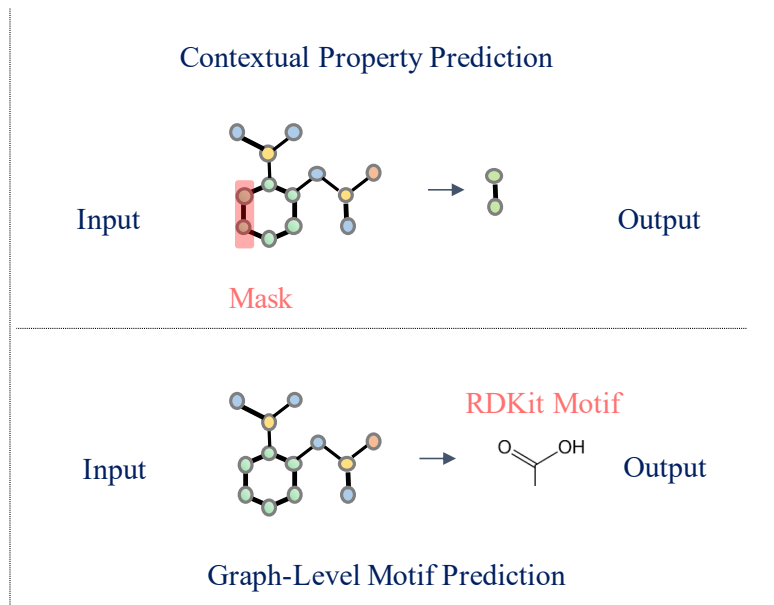

B. Context and Motif Prediction on Molecular Graphs

Figure 9: Illustrations of Self-Supervised Learning with Transformers.

Not unexpectedly, transformers are being actively applied in drug discovery. Notably, 
transformers enable effective self-supervised pretraining, such as masked language modeling (Fig 9A). In 2019, Wang et al 215 developed SMILES-BERT, which consists of several transformer encoder layers, to improve molecular property prediction. SMILES-BERT is first pretrained on a large-scale corpus of SMILES strings via a SMILES recovery task and then fine-tuned on the downstream prediction tasks. Later, Honda et al ${ }^{216}$ proposed to learn molecular representations through pretraining a sequence-to-sequence language model, which is termed as the SMILES Transformer. Chithrananda et al ${ }^{59}$ also developed ChemBERTa, built upon the RoBERTa model ${ }^{211}$ for molecular property prediction. More recently, Fabian et al ${ }^{95}$ applied the architecture of BERT ${ }^{209}$ to learn molecular representations, also referred to as MolBERT. When pretrained with masked language modeling and other tasks, MolBERT achieves improved performance for molecular property prediction compared to the fixed fingerprints. Moreover, transformers can also be applied on molecular graphs, especially

considering that the transformer encoder can be viewed as a GAT variant. 150 For example, Rong et al $\frac{63}{3}$ developed a novel framework, GROVER, to learn graph representations with the message passing transformer. By designing self-supervised contextual property prediction and graph-level motif prediction tasks (Fig 9B), GROVER is pretrained on 10 million unlabeled molecules and achieves state-of-the-art performance on 11 benchmark datasets.

In addition to molecular property prediction, transformers can also be exploited for molecule generation, such as MoleculeChef, $\frac{217}{2}$ which can generate the reactants for a given product, similar to machine translation. More recently, transformers are also exploited for protein-specific molecule generation, 218 where the input is the amino acid sequence of the target protein and the output are ligands in the SMILES representation.

\section{Learning Paradigms}

Drug discovery, despite the light shed by AI, still faces major challenges. For molecular property prediction, labeled data points are at the core of machine learning models. Nonetheless, 
in real-world settings, generating labeled data points in wet lab can be very expensive. Consequently, the datasets for model training are usually limited in size, exhibit high sparsity, and can be heavily biased and noisy, which is also termed as the low-data drug discovery problem. $\frac{158 \mid 165}{16}$ For molecule generation, although existing generative models, such as VAEs, can be used to generate molecules towards desired properties, the mechanism by mapping from the points in the latent space to real molecules which are most proximal can limit the

exploration of the chemical space, leading to low novelty and diversity. ${ }^{78}$ To address these challenges, various learning paradigms have been proposed. In this survey, we mainly focus on self-supervised learning and reinforcement learning to address molecular property prediction and molecule generation, respectively. Other learning paradigms are also discussed.

\section{Self-Supervised Learning}

The performance of deep neural networks, especially supervised learning, hinges on a large labeled dataset. Nevertheless, supervised learning is meeting its bottleneck due to its heavy reliance on expensive manually-labeled data. $\frac{219}{2 n}$ real-world problems such as molecular property prediction, the labeled data is often limited, sparse and biased, which leads to low generalizalibility of models. Self-supervised learning is promising paradigm and has achieved state-of-the-art performance in learning with limited labels, as adopted in the aforementioned language models, for instance, BERT. ${ }^{209}$ Notably, self-supervised learning should be distinguished from unsupervised learning. Unsupervised learning focuses on detecting patterns in data without labels, such as clustering, whereas self-supervised learning aims to recover the data. More specifically, it can be classified into two main types, i.e., generative and contrastive self-supervised learning.

For the generative self-supervised learning, a canonical task is the masked language modeling, as proposed in BERT, 209 where the model is trained to predict the masked tokens, thereby recovering the original input. Model parameterization is usually implemented by optimizing the cross-entropy loss between the output and the masked tokens in the in- 
put. Representative works of self-supervised learning in drug discovery are discussed in Section "Transformers" (e.g., MolBERT ${ }^{95}$ and GROVER ${ }^{63}$ ). Notably, self-supervised pretraining can avoid the negative transfer caused by supervised pretraining - transfer of knowledge from pretraining harms model generalization, as shown by $\mathrm{Hu}$ et al. $\frac{163}{16}$ Besides, contrastive learning is another type of self-supervised learning. More specifically, contrastive learning aims to learn latent representations through contrasting data pairs (positive vs negative), where the positive and negative examples are constructed by augmenting the unlabeled samples in a self-supervised manner. Recently, contrastive learning has been employed to address the low-data drug discovery problem. For instance, Wang et al ${ }^{220}$ proposed molecular contrastive learning of representations (MolCLR) on molecular graphs for molecular property prediction. Three molecular graph augmentation ways are used, i.e., atom masking, bond deletion, and subgraph removal. Through a contrastive loss, MolCLR learns molecular representations by contrasting positive vs negative molecules, where molecular graph pairs augmented from the same molecule are treated as the positive and the others denoted as negative. Experimental results show that MolCLR can effectively transfer the learned representations to downstream tasks and achieve state-of-the-art performance in molecular property prediction.

In addition to self-supervised learning, other learning paradigms have also been exploited to address the low-data drug discovery challenge. For example, meta learning ${ }^{221}$ aims to learn a learner to be adapted to new tasks. In a study by Nguyen et al, $\frac{165}{16}$ the meta-learning initializations outperform multi-task pretraining baselines on 16 out of 20 in-distribution tasks and all out-of-distribution tasks. A member of the meta-learning family is few-shot learning,$\stackrel{222}{2}$ the core idea of which is to generalize with a few examples. For instance, Altae Tran et al ${ }^{158}$ proposed a one-shot learning framework for activity classification, which lowers the amount of data required for predictions. Intuitively, through learning a distance metric, molecules can be embedded into the latent space in a more organized way. Thus, when new molecules come, their embeddings can be compared to the exiting labeled molecules for 
more accurate prediction. Closely to this idea, another paradigm is metric learning, 2231224 which mainly deals with data with mixed distribution due to the activity cliffs. $\frac{38}{}$ Metric learning has been widely applied in computer vision, $\underline{224225}$ especially in situations where the similarity or distance must be computed for clustering or nearest neighbor classification purpose. At its core, a distance metric (e.g., cosine distance) is to be learned, based on which the learned latent representations of the input data can be separated according to their labels. In a recent work by $\mathrm{Na}$ et al, 226 a generalized deep metric learning (GeDML) framework is proposed, which alleviates the structure-property mismatch problem through better separating molecules in the latent space. The representations learned via metric learning are also conducive for goal-directed molecule generation, i.e., search in the chemical space. For example, Koge et a ${ }^{[227}$ proposed a molecular embedding framework by combining VAEs and metric learning. The idea is to make the molecules' embedding in the latent space consistent with their properties, thereby enabling efficient search during molecule generation.

\section{Reinforcement Learning}

With improved performance on molecular property prediction, another challenge still poses for molecule generation, i.e., how to design molecules with the desired properties? As mentioned in Section "Model Architectures", VAEs and flow models can be used to generate molecules with preferred properties by sampling from a learned latent space. However, the latent space can be highly dimensional and the objective functions defined in the latent space is usually non-convex, making it difficult to optimize the properties of generated molecules. $\frac{169}{16}$ Consequently, reinforcement learning $(\mathrm{RL})$ is often used as the alternative to navigate the chemical space, which mainly deals with how an agent should take actions in a certain state so as to maximize a reward or return. ${ }^{228} \mathrm{RL}$ algorithms can be classified into 1) value-based (e.g., Q-learning), 2) policy-based (e.g., policy gradient) and 3) hybrid (e.g., actor-critic). 229

In drug discovery, the DMTA cycle (see Section "Drug Discovery Overview") underlying drug design, $\underline{3}$ i.e., goal-directed molecule generation, can be potentially automated 
by RL through connecting the generative model (i.e., the agent for molecule generation) and the predictive model (i.e., assigning rewards based on the predicted property values). For example, Zhou et al $\frac{169}{169}$ adopted value-based, double Q-learning (DQN) $)^{230}$ to optimize the generated molecules. Other related works mainly adopt the policy-gradient algorithm,

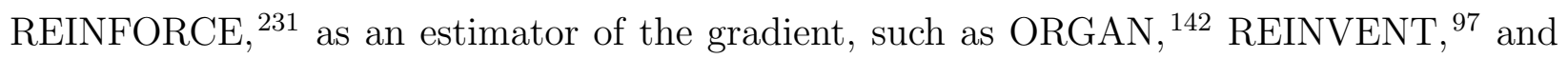
ORGANIC.143 Another policy-gradient algorithm, proximal policy optimization (PPO), 232 is also gaining popularity recently, which is improved from the trust region policy optimisation (TRPO). $\stackrel{233}{2}$ TRPO employs a trust region so that optimizations are restricted to a region where the approximation of the true cost function holds, thereby preventing policies updated too wildly and lowering the chance of a catastrophically "bad" update. $\frac{229}{}$ However, TRPO requires the calculation of second-order gradients, being computationally expensive. PPO, on the contrary, only requires first-order gradients and can retain the performance of TRPO, exhibiting low sample complexity. Studies adopting PPO for de novo drug design include the work by Neil et al, ${ }^{132}$ GCPN,,$\frac{140}{}$ DeepGraphMolGen $\frac{170}{170}$ and MNCE-RL. 171

Nevertheless, policy-gradient algorithms usually exhibit high variance since the gradient estimation can be noisy. $\stackrel{229}{2}$ To reduce the variance, an improved class of algorithms is the hybrid actor-critic method, which combines policy-gradient methods with learned value functions. For example, off-policy deterministic policy gradient (DPG) extends the standard policy gradients for stochastic policies to deterministic policies, which only integrates over the state space instead of both state and action spaces, thus requiring fewer samples in problems with large action spaces. Later, deep deterministic policy gradient (DDPG) utilizes neural networks on high-dimensional space is introduced, as adopted in MolGAN.197 Another technique for variance minimization to accelerate convergence is to subtract the estimated reward from the true reward, which separates the policy training from value estimation, also known as the advantage actor-critic (A2C) algorithm adopted by Neil et al.132

With RL training, chemical libraries shifted towards desired properties are expected to be generated. However, drug design is a multi-objective optimization problem. ${ }^{91234}$ In order 
to prioritize molecules based on the pre-defined goals, non-dominated sorting or pair-based comparisons are usually exploited to find solutions with the Pareto optimality. ${ }^{235} \sqrt{237}$ Another issue with RL is the trade-off between exploration and exploitation. As illustrated by Zhou et al, $\stackrel{169}{1}$ this trade-off is a dilemma underlying by uncertainty. Due to the lack of a complete knowledge of the rewards for all the states, if constantly choosing the best action known to produce the highest reward (exploitation), the model will never learn anything about the rewards of the other states; on the other hand, if always choosing a random action (exploration), the model will not receive sufficient reward. A potential solution for the exploration-exploitation trade-off is active learning, which is a paradigm where the model can query an expert or any other information sources in an active manner during learning. .11238

\section{Discussions}

There has been a surge of AI in drug discovery over the past decade, which is still gaining popularity. Nonetheless, there are still challenges to be addressed. Despite the prosperity of deep learning models, it should be emphasized that data is at the core of developing and evaluating the models. ${ }^{239240}$ To make the models (either predictive or generative) more useful, data must be in sufficient amount and should maintain high quality. However, a fact to our dismay is that although existing chemical libraries have a large amount of molecules, the number of data points for each specific assay can be very scarce. ${ }^{239}$ Sometimes, even the quality of benchmark datasets is questionable with regard to the representative power for real-world drug discovery imposed by the vast chemical space. ${ }^{[55}$ Datasets in drug discovery can be highly imbalanced. $\frac{58}{5}$ Thus, when evaluating the models, there is need to obtain appropriate datasets and also consider data balancing methods as well as proper evaluation metrics (e.g., AUPRC vs AUROC). ㅁ

Besides, for the DMTA cycle (see Section "Drug Discovery Overview") in drug design, it should always be driven by a need or certain hypotheses. ${ }^{9}$ Even equipped with perfect 
predictive and generative models, a question still remains, i.e., what are the hypotheses for designing a drug candidate? In other words, what are the desired properties underlying an ideal drug candidate? To generate the insights into drug design, real-world data (e.g., electronic health records (EHR) and marketed drug databases) ${ }^{241}$ is receiving substantial attention for understanding the effectiveness and side effects of different therapeutics. Recently, we mined a large-scale EHR database for the innate properties underlying opioid analgesics with reduced overdose effects. ${ }^{242}$ We also mined the DrugBank database to identify key pharmacological components (i.e., carriers, transporters, enzymes and targets) underlying drug-drug interactions (DDIs). ${ }^{243}$ These patterns emerging from real-world data (RWD) allows hypotheses generation and can calibrate drug design insights. ${ }^{241}$

Another challenge is that deep learning, despite its superior performance, still leaves the model elusive for human interpretation. An ongoing need is, therefore, to develop explainable models with high interpretability. More specifically, there are four aspects to cover: ${ }^{[11} 1$ ) Transparency, which is knowing how the system reaches a particular answer;2) Justification, which is elucidating why the answer provided by the model is acceptable;3) Informativeness, which is providing new information to human decision makers; and 4) Uncertainty estimation, which is quantifying how reliable a prediction is. An ideal state is that AI can allow scientists to hone their knowledge and beliefs on the investigated process. For more details on explainable AI in drug discovery, we refer the readers to the review by Jimenez-Luna et al. 31

In addition to the scientific challenges, technical concerns remain. One unignorable reality is that, even for the state-of-the-art representation learning on molecular graphs, fixed fingerprints can still outperform GNN-derived representations for molecular property prediction. ${ }^{[244}$ In fact, ECFPs are a component of some GNN models. ${ }^{[6371}$ Besides, there is a lack of a unified protocol for AI-driven drug discovery studies. For example, different benchmark datasets, different split folds and evaluation metrics are used across the studies for molecular property prediction, let alone the varying hyper-parameters tuning, training and evaluation 
procedure. ${ }^{244}$ For molecule generation, Walters et al ${ }^{239}$ already proposed a few guidelines to evaluate the novelty of AI-discovered molecules. Likewise, protocols for molecular property prediction are also needed.

Overall, there are many promising opportunities as well as significant challenges when applying AI in drug discovery. In order to launch successful applications, we need to understand the basic concepts and consider the task, the data, the molecule representation, the model architecture and the learning paradigm as a whole. In this survey, we have covered multiple aspects centered around AI-driven drug discovery. We envision that with these aspects well understood, more meaningful contributions will be made to substantially transform this field.

\section{Author contributions statement}

J.D. and Z.Y. conceived the manuscript and the GitHub repository. J.D. drafted the manuscript and built the GitHub repository. All authors made critical revisions and review on the manuscript.

\section{Acknowledgement}

This project is partially funded by a Stony Brook University OVPR Seed Grant. The neural networks templates are from Visuals by dair.ai (https://github.com/dair-ai/ml-visuals).

\section{Supporting Information Available}

https://github.com/dengjianyuan/Survey_AI_Drug_Discovery 


\section{References}

(1) Mullard, A. New drugs cost US $\$ 2.6$ billion to develop. Nat. Rev. Drug Discov. 2014, 13,877 .

(2) Dowden, H.; Munro, J. Trends in clinical success rates and therapeutic focus. Nat. Rev. Drug Discov. 2019, 18, 495-497.

(3) Schneider, G. Automating drug discovery. Nat. Rev. Drug Discov. 2018, 17, 97.

(4) Chen, H.; Engkvist, O.; Wang, Y.; Olivecrona, M.; Blaschke, T. The rise of deep learning in drug discovery. Drug Discov. Today 2018, 23, 1241-1250.

(5) Mater, A. C.; Coote, M. L. Deep learning in chemistry. J. Chem. Inf. Model 2019, $59,2545-2559$.

(6) Vamathevan, J.; Clark, D.; Czodrowski, P.; Dunham, I.; Ferran, E.; Lee, G.; Li, B.; Madabhushi, A.; Shah, P.; Spitzer, M., et al. Applications of machine learning in drug discovery and development. Nat. Rev. Drug Discov. 2019, 18, 463-477.

(7) Paul, D.; Sanap, G.; Shenoy, S.; Kalyane, D.; Kalia, K.; Tekade, R. K. Artificial intelligence in drug discovery and development. Drug Discovery Today 2021, 26, 80.

(8) Stumpfe, D.; Bajorath, J. Current trends, overlooked issues, and unmet challenges in virtual screening. J. Chem. Inf. Model 2020, 60, 4112-4115.

(9) Schneider, P.; Walters, W. P.; Plowright, A. T.; Sieroka, N.; Listgarten, J.; Goodnow, R. A.; Fisher, J.; Jansen, J. M.; Duca, J. S.; Rush, T. S., et al. Rethinking drug design in the artificial intelligence era. Nat. Rev. Drug Discov. 2020, 19, 353-364.

(10) Boström, J.; Brown, D. G.; Young, R. J.; Keserü, G. M. Expanding the medicinal chemistry synthetic toolbox. Nat. Rev. Drug Discov. 2018, 17, 709-727. 
(11) Strokach, A.; Becerra, D.; Corbi-Verge, C.; Perez-Riba, A.; Kim, P. M. Fast and flexible protein design using deep graph neural networks. Cell Syst. 2020, 11, 402411.

(12) Pushpakom, S.; Iorio, F.; Eyers, P. A.; Escott, K. J.; Hopper, S.; Wells, A.; Doig, A.; Guilliams, T.; Latimer, J.; McNamee, C., et al. Drug repurposing: progress, challenges and recommendations. Nat. Rev. Drug Discov. 2019, 18, 41-58.

(13) Tsigelny, I. F. Artificial intelligence in drug combination therapy. Brief. Bioinformatics 2019, 20, 1434-1448.

(14) Paananen, J.; Fortino, V. An omics perspective on drug target discovery platforms. Brief. Bioinformatics 2020, 21, 1937-1953.

(15) Hughes, J. P.; Rees, S.; Kalindjian, S. B.; Philpott, K. L. Principles of early drug discovery. Br. J. Pharmacol. 2011, 162, 1239-1249.

(16) Pereira, D.; Williams, J. Origin and evolution of high throughput screening. Br. J. Pharmacol. 2007, 152, 53-61.

(17) Bender, A.; Bojanic, D.; Davies, J. W.; Crisman, T. J.; Mikhailov, D.; Scheiber, J.; Jenkins, J. L.; Deng, Z.; Hill, W. A. G.; Popov, M., et al. Which aspects of HTS are empirically correlated with downstream success? Curr Opin Drug Discov Devel 2008, 11,327 .

(18) Wang, Y.; Bryant, S. H.; Cheng, T.; Wang, J.; Gindulyte, A.; Shoemaker, B. A.; Thiessen, P. A.; He, S.; Zhang, J. Pubchem bioassay: 2017 update. Nucleic Acids Res. 2017, 45, D955-D963.

(19) Sterling, T.; Irwin, J. J. ZINC 15-ligand discovery for everyone. J. Chem. Inf. Model 2015, 55, 2324-2337. 
(20) Kim, S. Getting the most out of PubChem for virtual screening. Expert Opin Drug Discov 2016, 11, 843-855.

(21) Scior, T.; Bender, A.; Tresadern, G.; Medina-Franco, J. L.; Martínez-Mayorga, K.; Langer, T.; Cuanalo-Contreras, K.; Agrafiotis, D. K. Recognizing pitfalls in virtual screening: a critical review. J. Chem. Inf. Model 2012, 52, 867-881.

(22) Salahudeen, M. S.; Nishtala, P. S. An overview of pharmacodynamic modelling, ligandbinding approach and its application in clinical practice. Saudi Pharm J 2017, 25, $165-175$.

(23) Hu, Y.; Bajorath, J. Compound promiscuity: what can we learn from current data? Drug Discov. Today 2013, 18, 644-650.

(24) Yusof, I.; Shah, F.; Hashimoto, T.; Segall, M. D.; Greene, N. Finding the rules for successful drug optimisation. Drug Discov. Today 2014, 19, 680-687.

(25) Nicolaou, C. A.; Brown, N. Multi-objective optimization methods in drug design. Drug Discov. Today: Technologies 2013, 10, e427-e435.

(26) Muratov, E. N.; Bajorath, J.; Sheridan, R. P.; Tetko, I. V.; Filimonov, D.; Poroikov, V.; Oprea, T. I.; Baskin, I. I.; Varnek, A.; Roitberg, A., et al. QSAR without borders. Chem. Soc. Rev. 2020, 49, 3525-3564.

(27) Schneider, G.; Fechner, U. Computer-based de novo design of drug-like molecules. Nat. Rev. Drug Discov. 2005, 4, 649-663.

(28) Dobson, C. M. Chemical space and biology. 2004.

(29) Sliwoski, G.; Kothiwale, S.; Meiler, J.; Lowe, E. W. Computational methods in drug discovery. Pharmacol. Rev. 2014, 66, 334-395.

(30) Van Drie, J. H. Computer-aided drug design: the next 20 years. J. Comput. Aided Mol. Des. 2007, 21, 591-601. 
(31) Jiménez-Luna, J.; Grisoni, F.; Schneider, G. Drug discovery with explainable artificial intelligence. Nat. Mach. Intell. 2020, 2, 573-584.

(32) Bajorath, J. Integration of virtual and high-throughput screening. Nat. Rev. Drug Discov. 2002, 1, 882-894.

(33) Schneider, G. Virtual screening: an endless staircase? Nat. Rev. Drug Discov. 2010, 9, 273-276.

(34) Polishchuk, P. Interpretation of quantitative structure-activity relationship models: past, present, and future. J. Chem. Inf. Model 2017, 57, 2618-2639.

(35) Sydow, D.; Burggraaff, L.; Szengel, A.; van Vlijmen, H. W.; IJzerman, A. P.; van Westen, G. J.; Volkamer, A. Advances and challenges in computational target prediction. J. Chem. Inf. Model 2019, 59, 1728-1742.

(36) Maggiora, G. On outliers and activity cliffs-why QSAR often disappoints. J Chem Inf Model 2006, 46, 1535-1535.

(37) Stumpfe, D.; Hu, Y.; Dimova, D.; Bajorath, J. Recent progress in understanding activity cliffs and their utility in medicinal chemistry: miniperspective. J. Med. Chem. 2014, 57, 18-28.

(38) Bajorath, J. Duality of activity cliffs in drug discovery. Expert Opin Drug Discov 2019, $14,517-520$.

(39) Ma, J.; Sheridan, R. P.; Liaw, A.; Dahl, G. E.; Svetnik, V. Deep neural nets as a method for quantitative structure-activity relationships. J. Chem. Inf. Model 2015, $55,263-274$.

(40) Lavecchia, A. Machine-learning approaches in drug discovery: methods and applications. Drug Discov. Today 2015, 20, 318-331. 
(41) Krizhevsky, A.; Sutskever, I.; Hinton, G. E. Imagenet classification with deep convolutional neural networks. Advances in Neural Information Processing Systems 2012, 25, 1097-1105.

(42) Alom, M. Z.; Taha, T. M.; Yakopcic, C.; Westberg, S.; Sidike, P.; Nasrin, M. S.; Van Esesn, B. C.; Awwal, A. A. S.; Asari, V. K. The history began from alexnet: A comprehensive survey on deep learning approaches. arXiv preprint arXiv:1803.01164 2018

(43) Öztürk, H.; Özgür, A.; Schwaller, P.; Laino, T.; Ozkirimli, E. Exploring chemical space using natural language processing methodologies for drug discovery. Drug Discov. Today 2020, 25, 689-705.

(44) Jiménez-Luna, J.; Grisoni, F.; Weskamp, N.; Schneider, G. Artificial intelligence in drug discovery: Recent advances and future perspectives. Expert Opin Drug Discov $2021,1-11$.

(45) Zhavoronkov, A.; Ivanenkov, Y. A.; Aliper, A.; Veselov, M. S.; Aladinskiy, V. A.; Aladinskaya, A. V.; Terentiev, V. A.; Polykovskiy, D. A.; Kuznetsov, M. D.; Asadulaev, A., et al. Deep learning enables rapid identification of potent DDR1 kinase inhibitors. Nat. Biotechnol. 2019, 37, 1038-1040.

(46) Stokes, J. M.; Yang, K.; Swanson, K.; Jin, W.; Cubillos-Ruiz, A.; Donghia, N. M.; MacNair, C. R.; French, S.; Carfrae, L. A.; Bloom-Ackermann, Z., et al. A deep learning approach to antibiotic discovery. Cell 2020, 180, 688-702.

(47) Chuang, K. V.; Gunsalus, L. M.; Keiser, M. J. Learning Molecular Representations for Medicinal Chemistry: Miniperspective. J. Med. Chem. 2020, 63, 8705-8722.

(48) Mayr, A.; Klambauer, G.; Unterthiner, T.; Hochreiter, S. DeepTox: toxicity prediction using deep learning. Frontiers in Environmental Science 2016, 3, 80. 
(49) Andrade, R. J.; Chalasani, N.; Björnsson, E. S.; Suzuki, A.; Kullak-Ublick, G. A.; Watkins, P. B.; Devarbhavi, H.; Merz, M.; Lucena, M. I.; Kaplowitz, N., et al. Druginduced liver injury. Nat. Rev. Dis. Primers 2019, 5, 1-22.

(50) Elton, D. C.; Boukouvalas, Z.; Fuge, M. D.; Chung, P. W. Deep learning for molecular design - a review of the state of the art. Mol. Syst. Des. Eng. 2019, 4, 828-849.

(51) Mercado, R.; Rastemo, T.; Lindelöf, E.; Klambauer, G.; Engkvist, O.; Chen, H.; Bjerrum, E. J. Practical Notes on Building Molecular Graph Generative Models. Applied AI Letters 2020,

(52) Schaduangrat, N.; Lampa, S.; Simeon, S.; Gleeson, M. P.; Spjuth, O.; Nantasenamat, C. Towards reproducible computational drug discovery. J. Cheminformatics 2020, 12, 9 .

(53) Bender, A.; Cortes-Ciriano, I. Artificial intelligence in drug discovery: what is realistic, what are illusions? Part 1: Ways to make an impact, and why we are not there yet. Drug Discov. Today 2020,

(54) Bender, A.; Cortes-Ciriano, I. Artificial intelligence in drug discovery: what is realistic, what are illusions? Part 2: a discussion of chemical and biological data used for AI in drug discovery. Drug Discov. Today 2021,

(55) Walters, W. P.; Barzilay, R. Critical assessment of AI in drug discovery. Expert Opin Drug Discov 2021, 1-11.

(56) Rifaioglu, A. S.; Atas, H.; Martin, M. J.; Cetin-Atalay, R.; Atalay, V.; Doğan, T. Recent applications of deep learning and machine intelligence on in silico drug discovery: methods, tools and databases. Brief. Bioinformatics 2019, 20, 1878-1912.

(57) Kim, S.; Chen, J.; Cheng, T.; Gindulyte, A.; He, J.; He, S.; Li, Q.; Shoemaker, B. A.; 
Thiessen, P. A.; Yu, B., et al. PubChem in 2021: new data content and improved web interfaces. Nucleic Acids Res. 2021, 49, D1388-D1395.

(58) Korkmaz, S. Deep learning-based imbalanced data classification for drug discovery. $J$. Chem. Inf. Model 2020, 60, 4180-4190.

(59) Chithrananda, S.; Grand, G.; Ramsundar, B. ChemBERTa: Large-Scale Self-Supervised Pretraining for Molecular Property Prediction. arXiv preprint arXiv:2010.09885 2020,

(60) Gaulton, A.; Hersey, A.; Nowotka, M.; Bento, A. P.; Chambers, J.; Mendez, D.; Mutowo, P.; Atkinson, F.; Bellis, L. J.; Cibrián-Uhalte, E., et al. The ChEMBL database in 2017. Nucleic Acids Res. 2017, 45, D945-D954.

(61) Davies, M.; Nowotka, M.; Papadatos, G.; Dedman, N.; Gaulton, A.; Atkinson, F.; Bellis, L.; Overington, J. P. ChEMBL web services: streamlining access to drug discovery data and utilities. Nucleic Acids Res. 2015, 43, W612-W620.

(62) Mayr, A.; Klambauer, G.; Unterthiner, T.; Steijaert, M.; Wegner, J. K.; Ceulemans, H.; Clevert, D.-A.; Hochreiter, S. Large-scale comparison of machine learning methods for drug target prediction on ChEMBL. Chem. Sci 2018, 9, 5441-5451.

(63) Rong, Y.; Bian, Y.; Xu, T.; Xie, W.; Wei, Y.; Huang, W.; Huang, J. Grover: Selfsupervised message passing transformer on large-scale molecular data. arXiv preprint arXiv:2007.02835 2020,

(64) Polykovskiy, D.; Zhebrak, A.; Sanchez-Lengeling, B.; Golovanov, S.; Tatanov, O.; Belyaev, S.; Kurbanov, R.; Artamonov, A.; Aladinskiy, V.; Veselov, M., et al. Molecular sets (MOSES): a benchmarking platform for molecular generation models. Front. Pharmacol. 2020, 11. 
(65) Lagarde, N.; Zagury, J.-F.; Montes, M. Benchmarking data sets for the evaluation of virtual ligand screening methods: review and perspectives. J. Chem. Inf. Model 2015, $55,1297-1307$.

(66) Chen, M.; Suzuki, A.; Thakkar, S.; Yu, K.; Hu, C.; Tong, W. DILIrank: the largest reference drug list ranked by the risk for developing drug-induced liver injury in humans. Drug Discov Today 2016, 21, 648-653.

(67) David, L.; Thakkar, A.; Mercado, R.; Engkvist, O. Molecular representations in AIdriven drug discovery: a review and practical guide. J. Cheminformatics 2020, 12, $1-22$.

(68) Morgan, H. L. The generation of a unique machine description for chemical structuresa technique developed at chemical abstracts service. J. Chem. Doc 1965, 5, 107-113.

(69) Subramanian, G.; Ramsundar, B.; Pande, V.; Denny, R. A. Computational modeling of $\beta$-secretase 1 (BACE-1) inhibitors using ligand based approaches. J. Chem. Inf. Model 2016, 56, 1936-1949.

(70) Zang, Q.; Mansouri, K.; Williams, A. J.; Judson, R. S.; Allen, D. G.; Casey, W. M.; Kleinstreuer, N. C. In silico prediction of physicochemical properties of environmental chemicals using molecular fingerprints and machine learning. J. Chem. Inf. Model 2017, 57, 36-49.

(71) Yang, K.; Swanson, K.; Jin, W.; Coley, C.; Eiden, P.; Gao, H.; Guzman-Perez, A.; Hopper, T.; Kelley, B.; Mathea, M., et al. Analyzing learned molecular representations for property prediction. J. Chem. Inf. Model 2019, 59, 3370-3388.

(72) Mercado, R.; Rastemo, T.; Lindelöf, E.; Klambauer, G.; Engkvist, O.; Chen, H.; Bjerrum, E. J. Graph Networks for Molecular Design. Mach. Learn.: Sci. Technol. 2020, 
(73) Jin, W.; Barzilay, R.; Jaakkola, T. Multi-objective molecule generation using interpretable substructures. International Conference on Machine Learning. 2020; pp 48494859.

(74) Weininger, D. SMILES, a chemical language and information system. 1. Introduction to methodology and encoding rules. J Chem Inform Comput Sci 1988, 28, 31-36.

(75) Weininger, D.; Weininger, A.; Weininger, J. L. SMILES. 2. Algorithm for generation of unique SMILES notation. J Chem Inform Comput Sci 1989, 29, 97-101.

(76) Bian, Y.; Xie, X.-Q. Generative chemistry: drug discovery with deep learning generative models. J. Mol. Model. 2021, 27, 1-18.

(77) Xiong, Z.; Wang, D.; Liu, X.; Zhong, F.; Wan, X.; Li, X.; Li, Z.; Luo, X.; Chen, K.; Jiang, H., et al. Pushing the boundaries of molecular representation for drug discovery with the graph attention mechanism. J. Med. Chem. 2019, 63, 8749-8760.

(78) Gómez-Bombarelli, R.; Wei, J. N.; Duvenaud, D.; Hernández-Lobato, J. M.; SánchezLengeling, B.; Sheberla, D.; Aguilera-Iparraguirre, J.; Hirzel, T. D.; Adams, R. P.; Aspuru-Guzik, A. Automatic chemical design using a data-driven continuous representation of molecules. ACS Cent. Sci. 2018, 4, 268-276.

(79) Popova, M.; Isayev, O.; Tropsha, A. Deep reinforcement learning for de novo drug design. Sci. Adv. 2018, 4, eaap7885.

(80) Ragoza, M.; Hochuli, J.; Idrobo, E.; Sunseri, J.; Koes, D. R. Protein-ligand scoring with convolutional neural networks. J. Chem. Inf. Model 2017, 57, 942-957.

(81) Jiménez, J.; Skalic, M.; Martinez-Rosell, G.; De Fabritiis, G. K deep: protein-ligand absolute binding affinity prediction via 3d-convolutional neural networks. J. Chem. Inf. Model 2018, 58, 287-296. 
(82) Lim, J.; Ryu, S.; Park, K.; Choe, Y. J.; Ham, J.; Kim, W. Y. Predicting drug-target interaction using a novel graph neural network with 3D structure-embedded graph representation. J. Chem. Inf. Model 2019, 59, 3981-3988.

(83) Hernandez, M.; Liang Gan, G.; Linvill, K.; Dukatz, C.; Feng, J.; Bhisetti, G. A quantum-inspired method for three-dimensional ligand-based virtual screening. $J$. Chem. Inf. Model 2019, 59, 4475-4485.

(84) Wu, K.; Wei, G.-W. Quantitative toxicity prediction using topology based multitask deep neural networks. J. Chem. Inf. Model 2018, 58, 520-531.

(85) Skalic, M.; Jiménez, J.; Sabbadin, D.; De Fabritiis, G. Shape-based generative modeling for de novo drug design. J. Chem. Inf. Model 2019, 59, 1205-1214.

(86) Simm, G.; Pinsler, R.; Hernández-Lobato, J. M. Reinforcement learning for molecular design guided by quantum mechanics. International Conference on Machine Learning. 2020; pp 8959-8969.

(87) Hemmerich, J.; Asilar, E.; Ecker, G. F. COVER: conformational oversampling as data augmentation for molecules. J. Cheminformatics 2020, 12, 1-12.

(88) Fernandez, M.; Ban, F.; Woo, G.; Hsing, M.; Yamazaki, T.; LeBlanc, E.; Rennie, P. S.; Welch, W. J.; Cherkasov, A. Toxic colors: the use of deep learning for predicting toxicity of compounds merely from their graphic images. J. Chem. Inf. Model 2018, $58,1533-1543$.

(89) Meyer, J. G.; Liu, S.; Miller, I. J.; Coon, J. J.; Gitter, A. Learning drug functions from chemical structures with convolutional neural networks and random forests. $J$. Chem. Inf. Model 2019, 59, 4438-4449.

(90) Cortés-Ciriano, I.; Bender, A. KekuleScope: prediction of cancer cell line sensitivity 
and compound potency using convolutional neural networks trained on compound images. J. Cheminformatics 2019, 11, 1-16.

(91) Rifaioglu, A. S.; Nalbat, E.; Atalay, V.; Martin, M. J.; Cetin-Atalay, R.; Doğan, T. DEEPScreen: high performance drug-target interaction prediction with convolutional neural networks using 2-D structural compound representations. Chem. Sci 2020, 11, $2531-2557$.

(92) Rajan, K.; Brinkhaus, H. O.; Sorokina, M.; Zielesny, A.; Steinbeck, C. DECIMERSegmentation: Automated extraction of chemical structure depictions from scientific literature. J. Cheminformatics 2021, 13, 1-9.

(93) Wu, Z.; Ramsundar, B.; Feinberg, E. N.; Gomes, J.; Geniesse, C.; Pappu, A. S.; Leswing, K.; Pande, V. MoleculeNet: a benchmark for molecular machine learning. Chem. Sci 2018, 9, 513-530.

(94) Ramsundar, B.; Eastman, P.; Walters, P.; Pande, V.; Leswing, K.; Wu, Z. Deep Learning for the Life Sciences; O’Reilly Media, 2019; https://www.amazon.com/ Deep-Learning-Life-Sciences-Microscopy/dp/1492039837.

(95) Fabian, B.; Edlich, T.; Gaspar, H.; Segler, M.; Meyers, J.; Fiscato, M.; Ahmed, M. Molecular representation learning with language models and domain-relevant auxiliary tasks. arXiv preprint arXiv:2011.13230 2020,

(96) Shen, W. X.; Zeng, X.; Zhu, F.; li Wang, Y.; Qin, C.; Tan, Y.; Jiang, Y. Y.; Chen, Y. Z. Out-of-the-box deep learning prediction of pharmaceutical properties by broadly learned knowledge-based molecular representations. Nat. Mach. Intell. 2021, 3, 334-343.

(97) Olivecrona, M.; Blaschke, T.; Engkvist, O.; Chen, H. Molecular de-novo design through deep reinforcement learning. J. Cheminformatics 2017, 9, 1-14. 
(98) Blaschke, T.; Arús-Pous, J.; Chen, H.; Margreitter, C.; Tyrchan, C.; Engkvist, O.; Papadopoulos, K.; Patronov, A. REINVENT 2.0: An AI Tool for De Novo Drug Design. J. Chem. Inf. Model 2020,

(99) Brown, N.; Fiscato, M.; Segler, M. H.; Vaucher, A. C. GuacaMol: benchmarking models for de novo molecular design. J. Chem. Inf. Model 2019, 59, 1096-1108.

(100) Segler, M. H.; Kogej, T.; Tyrchan, C.; Waller, M. P. Generating focused molecule libraries for drug discovery with recurrent neural networks. ACS Cent. Sci. 2018, 4, $120-131$.

(101) Walters, W. P.; Murcko, M. A. Prediction of 'drug-likeness'. Adv. Drug Deliv. Rev. 2002, 54, 255-271.

(102) Schneider, N.; Jäckels, C.; Andres, C.; Hutter, M. C. Gradual in silico filtering for druglike substances. J. Chem. Inf. Model 2008, 48, 613-628.

(103) Palmer, D. S.; O'Boyle, N. M.; Glen, R. C.; Mitchell, J. B. Random forest models to predict aqueous solubility. J. Chem. Inf. Model 2007, 47, 150-158.

(104) Schroeter, T.; Schwaighofer, A.; Mika, S.; Ter Laak, A.; Suelzle, D.; Ganzer, U.; Heinrich, N.; Müller, K.-R. Machine learning models for lipophilicity and their domain of applicability. Mol. Pharm. 2007, 4, 524-538.

(105) Hou, T.; Wang, J.; Li, Y. ADME evaluation in drug discovery. 8. The prediction of human intestinal absorption by a support vector machine. J. Chem. Inf. Model 2007, 47, 2408-2415.

(106) Tian, S.; Li, Y.; Wang, J.; Zhang, J.; Hou, T. ADME evaluation in drug discovery. 9. Prediction of oral bioavailability in humans based on molecular properties and structural fingerprints. Mol. Pharm. 2011, 8, 841-851. 
(107) Sakiyama, Y.; Yuki, H.; Moriya, T.; Hattori, K.; Suzuki, M.; Shimada, K.; Honma, T. Predicting human liver microsomal stability with machine learning techniques. J. Mol. Graph. Model. 2008, 26, 907-915.

(108) Vasanthanathan, P.; Taboureau, O.; Oostenbrink, C.; Vermeulen, N. P.; Olsen, L.; Jørgensen, F. S. Classification of cytochrome P450 1A2 inhibitors and noninhibitors by machine learning techniques. Drug Metab. Dispos 2009, 37, 658-664.

(109) Riddick, G.; Song, H.; Ahn, S.; Walling, J.; Borges-Rivera, D.; Zhang, W.; Fine, H. A. Predicting in vitro drug sensitivity using Random Forests. Bioinformatics 2011, 27, 220-224.

(110) Zhao, C.; Zhang, H.; Zhang, X.; Liu, M.; Hu, Z.; Fan, B. Application of support vector machine (SVM) for prediction toxic activity of different data sets. Toxicology 2006, 217, 105-119.

(111) Heikamp, K.; Bajorath, J. Support vector machines for drug discovery. Expert Opin Drug Discov 2014, 9, 93-104.

(112) Svetnik, V.; Liaw, A.; Tong, C.; Culberson, J. C.; Sheridan, R. P.; Feuston, B. P. Random forest: a classification and regression tool for compound classification and QSAR modeling. J Chem Inform Comput Sci 2003, 43, 1947-1958.

(113) Dahl, G. Deep learning how I did it: Merck 1st place interview. Online article available from http://blog. kaggle. com/2012/11/01/deep-learning-how-i-did-it-merck-1st-placeinterview 2012,

(114) LeCun, Y.; Bengio, Y.; Hinton, G. Deep learning. Nature 2015, 521, 436-444.

(115) Simm, J.; Klambauer, G.; Arany, A.; Steijaert, M.; Wegner, J. K.; Gustin, E.; Chupakhin, V.; Chong, Y. T.; Vialard, J.; Buijnsters, P., et al. Repurposing high- 
throughput image assays enables biological activity prediction for drug discovery. Cell Chem. Biol. 2018, 25, 611-618.

(116) Hofmarcher, M.; Rumetshofer, E.; Clevert, D.-A.; Hochreiter, S.; Klambauer, G. Accurate prediction of biological assays with high-throughput microscopy images and convolutional networks. J. Chem. Inf. Model 2019, 59, 1163-1171.

(117) Ramsundar, B.; Kearnes, S.; Riley, P.; Webster, D.; Konerding, D.; Pande, V. Massively multitask networks for drug discovery. arXiv preprint arXiv:1502.02072 2015,

(118) Duvenaud, D.; Maclaurin, D.; Aguilera-Iparraguirre, J.; Gómez-Bombarelli, R.; Hirzel, T.; Aspuru-Guzik, A.; Adams, R. P. Convolutional networks on graphs for learning molecular fingerprints. arXiv preprint arXiv:1509.09292 2015,

(119) Glen, R. C.; Bender, A.; Arnby, C. H.; Carlsson, L.; Boyer, S.; Smith, J. Circular fingerprints: flexible molecular descriptors with applications from physical chemistry to ADME. IDrugs 2006, 9, 199.

(120) Goh, G. B.; Siegel, C.; Vishnu, A.; Hodas, N. O.; Baker, N. Chemception: a deep neural network with minimal chemistry knowledge matches the performance of expertdeveloped QSAR/QSPR models. arXiv preprint arXiv:1706.06689 2017,

(121) Huang, G.; Liu, Z.; Van Der Maaten, L.; Weinberger, K. Q. Densely connected convolutional networks. Proceedings of the IEEE Conference on Computer Vision and Pattern Recognition. 2017; pp 4700-4708.

(122) He, K.; Zhang, X.; Ren, S.; Sun, J. Deep residual learning for image recognition. Proceedings of the IEEE Conference on Computer Vision and Pattern Recognition. 2016; pp 770-778.

(123) Simonyan, K.; Zisserman, A. Very deep convolutional networks for large-scale image recognition. arXiv preprint arXiv:1409.1556 2014, 
(124) Staker, J.; Marshall, K.; Abel, R.; McQuaw, C. M. Molecular structure extraction from documents using deep learning. J. Chem. Inf. Model 2019, 59, 1017-1029.

(125) Rajan, K.; Zielesny, A.; Steinbeck, C. DECIMER: towards deep learning for chemical image recognition. J. Cheminformatics 2020, 12, 1-9.

(126) Hossain, M. Z.; Sohel, F.; Shiratuddin, M. F.; Laga, H. A comprehensive survey of deep learning for image captioning. ACM Computing Surveys (CsUR) 2019, 51, 1-36.

(127) Mikolov, T.; Kombrink, S.; Burget, L.; Černockỳ, J.; Khudanpur, S. Extensions of recurrent neural network language model. 2011 IEEE International Conference on Acoustics, Speech and Signal Processing (ICASSP). 2011; pp 5528-5531.

(128) Boulanger-Lewandowski, N.; Bengio, Y.; Vincent, P. Modeling temporal dependencies in high-dimensional sequences: Application to polyphonic music generation and transcription. arXiv preprint arXiv:1206.6392 2012,

(129) Hochreiter, S.; Schmidhuber, J. Long short-term memory. Neural Comput. 1997, 9, 1735-1780.

(130) Chung, J.; Gulcehre, C.; Cho, K.; Bengio, Y. Empirical evaluation of gated recurrent neural networks on sequence modeling. arXiv preprint arXiv:1412.3555 2014,

(131) Goh, G. B.; Hodas, N. O.; Siegel, C.; Vishnu, A. Smiles2vec: An interpretable general-purpose deep neural network for predicting chemical properties. arXiv preprint arXiv:1712.02034 2017,

(132) Neil, D.; Segler, M.; Guasch, L.; Ahmed, M.; Plumbley, D.; Sellwood, M.; Brown, N. Exploring deep recurrent models with reinforcement learning for molecule design. Proceedings of The International Conference on Learning Representations. 2018.

(133) Joulin, A.; Mikolov, T. Inferring algorithmic patterns with stack-augmented recurrent nets. arXiv preprint arXiv:1503.01007 2015, 
(134) Ståhl, N.; Falkman, G.; Karlsson, A.; Mathiason, G.; Bostrom, J. Deep reinforcement learning for multiparameter optimization in de novo drug design. J. Chem. Inf. Model 2019, 59, 3166-3176.

(135) Zheng, S.; Yan, X.; Yang, Y.; Xu, J. Identifying structure-property relationships through SMILES syntax analysis with self-attention mechanism. J. Chem. Inf. Model 2019, 59, 914-923.

(136) You, J.; Ying, R.; Ren, X.; Hamilton, W.; Leskovec, J. Graphrnn: Generating realistic graphs with deep auto-regressive models. International Conference on Machine Learning. 2018; pp 5708-5717.

(137) Li, Y.; Vinyals, O.; Dyer, C.; Pascanu, R.; Battaglia, P. Learning deep generative models of graphs. arXiv preprint arXiv:1803.03324 2018,

(138) Li, Y.; Zhang, L.; Liu, Z. Multi-objective de novo drug design with conditional graph generative model. J. Cheminformatics 2018, 10, 1-24.

(139) Popova, M.; Shvets, M.; Oliva, J.; Isayev, O. MolecularRnN: Generating realistic molecular graphs with optimized properties. arXiv preprint arXiv:1905.13372 2019,

(140) You, J.; Liu, B.; Ying, R.; Pande, V.; Leskovec, J. Graph convolutional policy network for goal-directed molecular graph generation. arXiv preprint arXiv:1806.02473 2018,

(141) Sattarov, B.; Baskin, I. I.; Horvath, D.; Marcou, G.; Bjerrum, E. J.; Varnek, A. De novo molecular design by combining deep autoencoder recurrent neural networks with generative topographic mapping. J. Chem. Inf. Model 2019, 59, 1182-1196.

(142) Guimaraes, G. L.; Sanchez-Lengeling, B.; Outeiral, C.; Farias, P. L. C.; AspuruGuzik, A. Objective-reinforced generative adversarial networks (ORGAN) for sequence generation models. arXiv preprint arXiv:1705.10843 2017, 
(143) Sanchez-Lengeling, B.; Outeiral, C.; Guimaraes, G. L.; Aspuru-Guzik, A. Optimizing distributions over molecular space. An objective-reinforced generative adversarial network for inverse-design chemistry (ORGANIC). ChemRxiv 2017, 2017.

(144) Wu, Z.; Pan, S.; Chen, F.; Long, G.; Zhang, C.; Philip, S. Y. A comprehensive survey on graph neural networks. IEEE Trans Neural Netw Learn $\mathbf{2 0 2 0 ,}$

(145) Li, Y.; Tarlow, D.; Brockschmidt, M.; Zemel, R. Gated graph sequence neural networks. arXiv preprint arXiv:1511.05493 2015,

(146) Defferrard, M.; Bresson, X.; Vandergheynst, P. Convolutional neural networks on graphs with fast localized spectral filtering. arXiv preprint arXiv:1606.09375 2016,

(147) Kipf, T. N.; Welling, M. Semi-supervised classification with graph convolutional networks. arXiv preprint arXiv:1609.02907 2016,

(148) Gilmer, J.; Schoenholz, S. S.; Riley, P. F.; Vinyals, O.; Dahl, G. E. Neural message passing for quantum chemistry. International Conference on Machine Learning. 2017; pp $1263-1272$.

(149) Hamilton, W. L.; Ying, R.; Leskovec, J. Inductive representation learning on large graphs. arXiv preprint arXiv:1706.02216 2017,

(150) Veličković, P.; Cucurull, G.; Casanova, A.; Romero, A.; Lio, P.; Bengio, Y. Graph attention networks. arXiv preprint arXiv:1710.10903 2017,

(151) Xu, K.; Hu, W.; Leskovec, J.; Jegelka, S. How powerful are graph neural networks? arXiv preprint arXiv:1810.00826 2018,

(152) Kearnes, S.; McCloskey, K.; Berndl, M.; Pande, V.; Riley, P. Molecular graph convolutions: moving beyond fingerprints. J. Comput. Aided Mol. Des. 2016, 30, 595-608.

(153) Landrum, G. RDKit: Open-Source Cheminformatics Software. RDKit 2016, 
(154) Withnall, M.; Lindelöf, E.; Engkvist, O.; Chen, H. Building attention and edge message passing neural networks for bioactivity and physical-chemical property prediction. J. Cheminformatics 2020, 12, 1-18.

(155) Schütt, K. T.; Kindermans, P.-J.; Sauceda, H. E.; Chmiela, S.; Tkatchenko, A.; Müller, K.-R. Schnet: A continuous-filter convolutional neural network for modeling quantum interactions. arXiv preprint arXiv:1706.08566 2017,

(156) Feinberg, E. N.; Sur, D.; Wu, Z.; Husic, B. E.; Mai, H.; Li, Y.; Sun, S.; Yang, J.; Ramsundar, B.; Pande, V. S. PotentialNet for molecular property prediction. ACS Cent. Sci. 2018, 4, 1520-1530.

(157) Klicpera, J.; Groß, J.; Günnemann, S. Directional message passing for molecular graphs. arXiv preprint arXiv:2003.03123 2020,

(158) Altae-Tran, H.; Ramsundar, B.; Pappu, A. S.; Pande, V. Low data drug discovery with one-shot learning. ACS Cent. Sci. 2017, 3, 283-293.

(159) Liu, S.; Demirel, M. F.; Liang, Y. N-gram graph: Simple unsupervised representation for graphs, with applications to molecules. arXiv preprint arXiv:1806.09206 2018,

(160) Lu, C.; Liu, Q.; Wang, C.; Huang, Z.; Lin, P.; He, L. Molecular property prediction: A multilevel quantum interactions modeling perspective. Proceedings of the AAAI Conference on Artificial Intelligence. 2019; pp 1052-1060.

(161) Cai, C.; Guo, P.; Zhou, Y.; Zhou, J.; Wang, Q.; Zhang, F.; Fang, J.; Cheng, F. Deep learning-based prediction of drug-induced cardiotoxicity. J. Chem. Inf. Model 2019, 59, 1073-1084.

(162) Wang, X.; Li, Z.; Jiang, M.; Wang, S.; Zhang, S.; Wei, Z. Molecule property prediction based on spatial graph embedding. J. Chem. Inf. Model 2019, 59, 3817-3828. 
(163) Hu, W.; Liu, B.; Gomes, J.; Zitnik, M.; Liang, P.; Pande, V.; Leskovec, J. Strategies for pre-training graph neural networks. arXiv preprint arXiv:1905.12265 2019,

(164) Hao, Z.; Lu, C.; Huang, Z.; Wang, H.; Hu, Z.; Liu, Q.; Chen, E.; Lee, C. ASGN: An active semi-supervised graph neural network for molecular property prediction. Proceedings of the 26th ACM SIGKDD International Conference on Knowledge Discovery \& Data Mining. 2020; pp 731-752.

(165) Nguyen, C. Q.; Kreatsoulas, C.; Branson, K. M. Meta-Learning Initializations for Low-Resource Drug Discovery. arXiv preprint arXiv:2003.05996 2020,

(166) Li, X.; Yan, X.; Gu, Q.; Zhou, H.; Wu, D.; Xu, J. DeepChemStable: Chemical stability prediction with an attention-based graph convolution network. J. Chem. Inf. Model 2019, 59, 1044-1049.

(167) Tang, B.; Kramer, S. T.; Fang, M.; Qiu, Y.; Wu, Z.; Xu, D. A self-attention based message passing neural network for predicting molecular lipophilicity and aqueous solubility. J. Cheminformatics 2020, 12, 1-9.

(168) Pathak, Y.; Laghuvarapu, S.; Mehta, S.; Priyakumar, U. D. Chemically interpretable graph interaction network for prediction of pharmacokinetic properties of drug-like molecules. Proceedings of the AAAI Conference on Artificial Intelligence. 2020; pp 873-880.

(169) Zhou, Z.; Kearnes, S.; Li, L.; Zare, R. N.; Riley, P. Optimization of molecules via deep reinforcement learning. Scientific reports 2019, 9, 1-10.

(170) Khemchandani, Y.; O’Hagan, S.; Samanta, S.; Swainston, N.; Roberts, T. J.; Bollegala, D.; Kell, D. B. DeepGraphMolGen, a multi-objective, computational strategy for generating molecules with desirable properties: a graph convolution and reinforcement learning approach. J. Cheminformatics 2020, 12, 1-17. 
(171) Xu, C.; Liu, Q.; Huang, M.; Jiang, T. Reinforced Molecular Optimization with Neighborhood-Controlled Grammars. arXiv preprint arXiv:2011.07225 2020,

(172) Kingma, D. P.; Welling, M. Auto-encoding variational bayes. arXiv preprint arXiv:1312.6114 2013,

(173) Kingma, D. P.; Welling, M. An introduction to variational autoencoders. arXiv preprint arXiv:1906.02691 2019,

(174) Kusner, M. J.; Paige, B.; Hernández-Lobato, J. M. Grammar variational autoencoder. International Conference on Machine Learning. 2017; pp 1945-1954.

(175) Dai, H.; Tian, Y.; Dai, B.; Skiena, S.; Song, L. Syntax-directed variational autoencoder for structured data. arXiv preprint arXiv:1802.08786 2018,

(176) Kang, S.; Cho, K. Conditional molecular design with deep generative models. J. Chem. Inf. Model 2018, 59, 43-52.

(177) Lim, J.; Ryu, S.; Kim, J. W.; Kim, W. Y. Molecular generative model based on conditional variational autoencoder for de novo molecular design. J. Cheminformatics 2018, 10, 1-9.

(178) Liu, Q.; Allamanis, M.; Brockschmidt, M.; Gaunt, A. L. Constrained graph variational autoencoders for molecule design. arXiv preprint arXiv:1805.09076 2018,

(179) Samanta, B.; De, A.; Jana, G.; Gómez, V.; Chattaraj, P. K.; Ganguly, N.; GomezRodriguez, M. Nevae: A deep generative model for molecular graphs. J Mach Learn Res 2020,

(180) Chenthamarakshan, V.; Das, P.; Padhi, I.; Strobelt, H.; Lim, K. W.; Hoover, B.; Hoffman, S. C.; Mojsilovic, A. Target-specific and selective drug design for covid-19 using deep generative models. arXiv preprint arXiv:2004.01215 2020, 
(181) Makhzani, A.; Shlens, J.; Jaitly, N.; Goodfellow, I.; Frey, B. Adversarial autoencoders. arXiv preprint arXiv:1511.05644 2015,

(182) Kadurin, A.; Nikolenko, S.; Khrabrov, K.; Aliper, A.; Zhavoronkov, A. druGAN: an advanced generative adversarial autoencoder model for de novo generation of new molecules with desired molecular properties in silico. Mol. Pharm. 2017, 14, 30983104.

(183) Blaschke, T.; Olivecrona, M.; Engkvist, O.; Bajorath, J.; Chen, H. Application of generative autoencoder in de novo molecular design. Mol. Inform. 2018, 37, 1700123.

(184) Polykovskiy, D.; Zhebrak, A.; Vetrov, D.; Ivanenkov, Y.; Aladinskiy, V.; Mamoshina, P.; Bozdaganyan, M.; Aliper, A.; Zhavoronkov, A.; Kadurin, A. Entangled conditional adversarial autoencoder for de novo drug discovery. Mol. Pharm. 2018, 15, 4398-4405.

(185) Simonovsky, M.; Komodakis, N. Graphvae: Towards generation of small graphs using variational autoencoders. International Conference on Artificial Neural Networks. 2018; pp 412-422.

(186) Jin, W.; Barzilay, R.; Jaakkola, T. Junction tree variational autoencoder for molecular graph generation. International Conference on Machine Learning. 2018; pp 2323-2332.

(187) Ma, T.; Chen, J.; Xiao, C. Constrained generation of semantically valid graphs via regularizing variational autoencoders. arXiv preprint arXiv:1809.02630 2018,

(188) Kajino, H. Molecular hypergraph grammar with its application to molecular optimization. International Conference on Machine Learning. 2019; pp 3183-3191.

(189) Kwon, Y.; Yoo, J.; Choi, Y.-S.; Son, W.-J.; Lee, D.; Kang, S. Efficient learning of non-autoregressive graph variational autoencoders for molecular graph generation. $J$. Cheminformatics 2019, 11, 1-10. 
(190) Lim, J.; Hwang, S.-Y.; Kim, S.; Moon, S.; Kim, W. Y. Scaffold-based molecular design using graph generative model. arXiv preprint arXiv:1905.13639 2019,

(191) Fu, T.; Xiao, C.; Sun, J. Core: Automatic molecule optimization using copy \& refine strategy. Proceedings of the AAAI Conference on Artificial Intelligence. 2020; pp 638645.

(192) Kwon, Y.; Lee, D.; Choi, Y.-S.; Shin, K.; Kang, S. Compressed graph representation for scalable molecular graph generation. J. Cheminformatics 2020, 12, 1-8.

(193) Jin, W.; Barzilay, R.; Jaakkola, T. Hierarchical generation of molecular graphs using structural motifs. International Conference on Machine Learning. 2020; pp 4839-4848.

(194) Goodfellow, I. J.; Pouget-Abadie, J.; Mirza, M.; Xu, B.; Warde-Farley, D.; Ozair, S.; Courville, A.; Bengio, Y. Generative adversarial networks. arXiv preprint $\operatorname{arXiv:1406.26612014,}$

(195) Yu, L.; Zhang, W.; Wang, J.; Yu, Y. Seqgan: Sequence generative adversarial nets with policy gradient. Proceedings of the AAAI Conference on Artificial Intelligence. 2017.

(196) Putin, E.; Asadulaev, A.; Ivanenkov, Y.; Aladinskiy, V.; Sanchez-Lengeling, B.; Aspuru-Guzik, A.; Zhavoronkov, A. Reinforced adversarial neural computer for de novo molecular design. J. Chem. Inf. Model 2018, 58, 1194-1204.

(197) De Cao, N.; Kipf, T. MolGAN: An implicit generative model for small molecular graphs. arXiv preprint arXiv:1805.11973 2018,

(198) Salimans, T.; Goodfellow, I.; Zaremba, W.; Cheung, V.; Radford, A.; Chen, X. Improved techniques for training gans. arXiv preprint arXiv:1606.03498 2016,

(199) Kobyzev, I.; Prince, S.; Brubaker, M. Normalizing flows: An introduction and review of current methods. IEEE PAMI 2020, 
(200) Dinh, L.; Krueger, D.; Bengio, Y. Nice: Non-linear independent components estimation. arXiv preprint arXiv:1410.8516 2014,

(201) Dinh, L.; Sohl-Dickstein, J.; Bengio, S. Density estimation using real nvp. arXiv preprint arXiv:1605.08803 2016,

(202) Madhawa, K.; Ishiguro, K.; Nakago, K.; Abe, M. Graphnvp: An invertible flow model for generating molecular graphs. arXiv preprint arXiv:1905.11600 2019,

(203) Honda, S.; Akita, H.; Ishiguro, K.; Nakanishi, T.; Oono, K. Graph residual flow for molecular graph generation. arXiv preprint arXiv:1909.13521 2019,

(204) Shi, C.; Xu, M.; Zhu, Z.; Zhang, W.; Zhang, M.; Tang, J. Graphaf: a flow-based autoregressive model for molecular graph generation. arXiv preprint arXiv:2001.09382 2020

(205) Zang, C.; Wang, F. MoFlow: an invertible flow model for generating molecular graphs. Proceedings of the 26th ACM SIGKDD International Conference on Knowledge Discovery \& Data Mining. 2020; pp 617-626.

(206) Luo, Y.; Yan, K.; Ji, S. GraphDF: A Discrete Flow Model for Molecular Graph Generation. arXiv preprint arXiv:2102.01189 2021,

(207) Vaswani, A.; Shazeer, N.; Parmar, N.; Uszkoreit, J.; Jones, L.; Gomez, A. N.; Kaiser, L.; Polosukhin, I. Attention is all you need. arXiv preprint arXiv:1706.03762 2017

(208) Radford, A.; Narasimhan, K.; Salimans, T.; Sutskever, I. Improving language understanding by generative pre-training. OpenAI 2018,

(209) Devlin, J.; Chang, M.-W.; Lee, K.; Toutanova, K. Bert: Pre-training of deep bidirectional transformers for language understanding. arXiv preprint arXiv:1810.04805 2018 
(210) Radford, A.; Wu, J.; Child, R.; Luan, D.; Amodei, D.; Sutskever, I. Language models are unsupervised multitask learners. OpenAI blog 2019, 1, 9.

(211) Liu, Y.; Ott, M.; Goyal, N.; Du, J.; Joshi, M.; Chen, D.; Levy, O.; Lewis, M.; Zettlemoyer, L.; Stoyanov, V. Roberta: A robustly optimized bert pretraining approach. arXiv preprint arXiv:190\%.11692 2019,

(212) Brown, T. B.; Mann, B.; Ryder, N.; Subbiah, M.; Kaplan, J.; Dhariwal, P.; Neelakantan, A.; Shyam, P.; Sastry, G.; Askell, A., et al. Language models are few-shot learners. arXiv preprint arXiv:2005.14165 2020,

(213) Carion, N.; Massa, F.; Synnaeve, G.; Usunier, N.; Kirillov, A.; Zagoruyko, S. End-toend object detection with transformers. European Conference on Computer Vision. 2020; pp 213-229.

(214) Dosovitskiy, A.; Beyer, L.; Kolesnikov, A.; Weissenborn, D.; Zhai, X.; Unterthiner, T.; Dehghani, M.; Minderer, M.; Heigold, G.; Gelly, S., et al. An image is worth 16x16 words: Transformers for image recognition at scale. arXiv preprint arXiv:2010.11929 2020 ,

(215) Wang, S.; Guo, Y.; Wang, Y.; Sun, H.; Huang, J. SMILES-BERT: large scale unsupervised pre-training for molecular property prediction. Proceedings of the 10th ACM International Conference on Bioinformatics, Computational Biology and Health Informatics. 2019; pp 429-436.

(216) Honda, S.; Shi, S.; Ueda, H. R. SMILES transformer: pre-trained molecular fingerprint for low data drug discovery. arXiv preprint arXiv:1911.04738 2019,

(217) Bradshaw, J.; Paige, B.; Kusner, M. J.; Segler, M. H.; Hernández-Lobato, J. M. A model to search for synthesizable molecules. arXiv preprint arXiv:1906.05221 2019, 
(218) Grechishnikova, D. Transformer neural network for protein-specific de novo drug generation as a machine translation problem. Scientific reports 2021, 11, 1-13.

(219) Liu, X.; Zhang, F.; Hou, Z.; Wang, Z.; Mian, L.; Zhang, J.; Tang, J. Self-supervised learning: Generative or contrastive. arXiv preprint arXiv:2006.08218 2020, 1.

(220) Wang, Y.; Wang, J.; Cao, Z.; Farimani, A. B. MolCLR: Molecular contrastive learning of representations via graph neural networks. arXiv preprint arXiv:2102.10056 2021,

(221) Vanschoren, J. Meta-learning: A survey. arXiv preprint arXiv:1810.03548 2018,

(222) Wang, Y.; Yao, Q.; Kwok, J. T.; Ni, L. M. Generalizing from a few examples: A survey on few-shot learning. ACM Computing Surveys (CSUR) 2020, 53, 1-34.

(223) Kulis, B., et al. Metric learning: A survey. Found. Trends Mach. Learn. 2012, 5, $287-364$.

(224) Yang, Z.; Bastan, M.; Zhu, X.; Gray, D.; Samaras, D. Hierarchical Proxy-based Loss for Deep Metric Learning. arXiv preprint arXiv:2103.13538 2021,

(225) Movshovitz-Attias, Y.; Toshev, A.; Leung, T. K.; Ioffe, S.; Singh, S. No fuss distance metric learning using proxies. Proceedings of the IEEE International Conference on Computer Vision. 2017; pp 360-368.

(226) Na, G. S.; Chang, H.; Kim, H. W. Machine-guided representation for accurate graphbased molecular machine learning. Phys. Chem. Chem. Phys. 2020, 22, 18526-18535.

(227) Koge, D.; Ono, N.; Huang, M.; Altaf-Ul-Amin, M.; Kanaya, S. Embedding of Molecular Structure Using Molecular Hypergraph Variational Autoencoder with Metric Learning. Mol. Inform. 2020,

(228) Sutton, R. S.; Barto, A. G. Reinforcement learning: An introduction; MIT press, 2018. 
(229) Arulkumaran, K.; Deisenroth, M. P.; Brundage, M.; Bharath, A. A. A brief survey of deep reinforcement learning. arXiv preprint arXiv:1708.05866 2017,

(230) Van Hasselt, H.; Guez, A.; Silver, D. Deep reinforcement learning with double qlearning. Proceedings of the AAAI Conference on Artificial Intelligence. 2016.

(231) Williams, R. J. Simple statistical gradient-following algorithms for connectionist reinforcement learning. Machine learning 1992, 8, 229-256.

(232) Schulman, J.; Wolski, F.; Dhariwal, P.; Radford, A.; Klimov, O. Proximal policy optimization algorithms. arXiv preprint arXiv:170\%.06347 2017,

(233) Schulman, J.; Levine, S.; Abbeel, P.; Jordan, M.; Moritz, P. Trust region policy optimization. International Conference on Machine Learning. 2015; pp 1889-1897.

(234) Deng, J.; Yang, Z.; Li, Y.; Samaras, D.; Wang, F. Towards Better Opioid Antagonists Using Deep Reinforcement Learning. arXiv preprint arXiv:2004.04768 2020,

(235) Yasonik, J. Multiobjective de novo drug design with recurrent neural networks and nondominated sorting. J. Cheminformatics 2020, 12, 1-9.

(236) Domenico, A.; Nicola, G.; Daniela, T.; Fulvio, C.; Nicola, A.; Orazio, N. De novo drug design of targeted chemical libraries based on artificial intelligence and pair-based multiobjective optimization. J. Chem. Inf. Model 2020, 60, 4582-4593.

(237) Liu, X.; Ye, K.; Van Vlijmen, H.; Emmerich, M.; IJzerman, A. P.; van Westen, G. DrugEx v2: De Novo Design of Drug Molecule by Pareto-based Multi-Objective Reinforcement Learning in Polypharmacology. ChemRxiv.14474127.v2 2021,

(238) Reker, D.; Schneider, G. Active-learning strategies in computer-assisted drug discovery. Drug Discov. Today 2015, 20, 458-465.

(239) Walters, W. P.; Murcko, M. Assessing the impact of generative AI on medicinal chemistry. Nat. Biotechnol. 2020, 38, 143-145. 
(240) Sambasivan, N.; Kapania, S.; Highfill, H.; Akrong, D.; Paritosh, P.; Aroyo, L. M. "Everyone wants to do the model work, not the data work": Data Cascades in HighStakes AI. proceedings of the 2021 CHI Conference on Human Factors in Computing Systems. 2021; pp 1-15.

(241) Singh, G.; Schulthess, D.; Hughes, N.; Vannieuwenhuyse, B.; Kalra, D. Real world big data for clinical research and drug development. Drug Discov. Today 2018, 23, $652-660$.

(242) Deng, J.; Hou, W.; Dong, X.; Hajagos, J.; Saltz, M.; Saltz, J.; Wang, F. A Large-Scale Observational Study on the Temporal Trends and Risk Factors of Opioid Overdose: Real-World Evidence for Better Opioids. Drugs-Real World Outcomes 2021, 1-14.

(243) Deng, J.; Wang, F. An Informatics-based Approach to Identify Key Pharmacological Components in Drug-Drug Interactions. AMIA Jt Summits Transl Sci Proc 2020, 2020, 142 .

(244) Jiang, D.; Wu, Z.; Hsieh, C.-Y.; Chen, G.; Liao, B.; Wang, Z.; Shen, C.; Cao, D.; Wu, J.; Hou, T. Could graph neural networks learn better molecular representation for drug discovery? A comparison study of descriptor-based and graph-based models. J. Cheminformatics 2021, 13, 1-23. 


\title{
Graphical TOC Entry
}

\begin{abstract}
Some journals require a graphical entry for the Table of Contents. This should be laid out "print ready" so that the sizing of the text is correct. Inside the tocentry environment, the font used is Helvetica $8 \mathrm{pt}$, as required by Journal of the American Chemical Society.

The surrounding frame is $9 \mathrm{~cm}$ by $3.5 \mathrm{~cm}$, which is the maximum permitted for Journal of the American Chemical Society graphical table of content entries. The box will not resize if the content is too big: instead it will overflow the edge of the box.

This box and the associated title will always be printed on a separate page at the end of the document.
\end{abstract}

